\title{
Relaxed constraints on the heavy scalar masses in 2HDM
}

\author{
Siddhartha Karmakar ${ }^{*}$ and Subhendu Rakshit ${ }^{\dagger}$ \\ Discipline of Physics, Indian Institute of Technology Indore, Khandwa Road, \\ Simrol, Indore-453 552, India
}

(Received 11 April 2019; published 16 September 2019)

\begin{abstract}
In the wake of new scalar searches at the LHC in various channels, it is interesting to investigate the sacrosanctity of the constraints on the masses and couplings of the heavier scalars in a two-Higgs-doublet model (2HDM). We consider the effects of new physics beyond a 2HDM encoded in terms of bosonic dim- 6 operators. Although these constraints are mostly immune to such new physics, we demonstrate that, for a specific class of bosonic operators, the constraints on the masses of the exotic scalars from cascade decays can get substantially relaxed. We present such effects for both degenerate and hierarchical mass spectra of the heavier scalars in 2HDM. Some decay channels of the new scalars vanish at the alignment limit in the tree-level 2HDM. But the inclusion of dim-6 terms can lead to significant cross sections for such processes. It is also pointed out that the observation of such processes can no longer rule out the alignment limit if such dim-6 operators are present.
\end{abstract}

DOI: 10.1103/PhysRevD.100.055016

\section{INTRODUCTION}

Even after the discovery of a Higgs boson [1,2] whose characteristics resemble that of the standard model (SM) Higgs, the dynamics of electroweak symmetry breaking and the structure of the scalar sector remains an open question. The noncancellation of quadratic divergence of Higgs mass under the framework of SM has motivated a plethora of beyond-standard model (BSM) theories for decades. The two-Higgs-doublet model (2HDM) is an archetype of an extended scalar sector, theoretically well motivated from the viewpoint of supersymmetry, composite Higgs models, etc. For example, in supersymmetric models [3] the motivation behind a second Higgs doublet is twofold: first to cancel chiral anomalies created by the superpartners of such scalars and second from the requirement of the superpotential to be holomorphic. 2HDMs arising in the framework of composite Higgs [4,5], little Higgs [6], and twin Higgs [7] have also been studied in the literature. Even keeping the hierarchy problem aside, it is often deployed to explain issues of electroweak baryogenesis $[8,9]$, flavor anomalies $[10,11]$, neutrino mass $[12,13]$, dark matter [14], etc.

In light of measurements of the signal strengths of the observed Higgs, any model with a scalar sector beyond the

\footnotetext{
phd1401251010@iiti.ac.in

†rakshit@iiti.ac.in
}

Published by the American Physical Society under the terms of the Creative Commons Attribution 4.0 International license. Further distribution of this work must maintain attribution to the author(s) and the published article's title, journal citation, and DOI. Funded by SCOAP ${ }^{3}$.
SM must contain a $C P$-even neutral scalar whose couplings are aligned to that of the SM Higgs boson. Such an alignment can be realized when the new scalars which mix with the SM-like Higgs are decoupled from the mass spectrum of SM à la Applequist-Carrazone [15,16]. The "alignment without decoupling" scenario becomes viable only for models with additional scalar doublets [16-22]. In such cases, the scalars can have masses below a TeV, i.e., well within the reach of the LHC. Thus, along with the signal strengths of the SM-like Higgs, the direct bounds on the masses of exotic scalars also play a pivotal role in constraining the parameter space of the 2HDM. Such bounds also depend on the specifications of the Yukawa sector of the models. Nonobservation of such new scalars rules out a significant region of parameter space in the alignment without decoupling scenarios. Also, some decay channels involving exotic scalars remain absent at the alignment limit in a $C P$-conserving $2 \mathrm{HDM}$ [23]. If the LHC discovers any new scalar state in one of these channels, the interpretation involving a $C P$-conserving $2 \mathrm{HDM}$ would readily imply a deviation from the alignment limit.

If new physics beyond 2HDM exists as a decoupled sector from the mass scale of the 2HDM, the effects of such new physics can be encoded in the higher-dimensional operators in an effective theory where the fields of the 2HDM constitute the low-energy spectrum [24-26]. Such an effective theory is dubbed as the two-Higgs-doublet model effective field theory (2HDMEFT) in the literature. Several aspects of such effective theories for various extended scalar sectors have been addressed in the literature [27-31]. A complete basis of the 6-dim operators in 2HDMEFT has been introduced only recently [26]. It has 
also been shown that such 6-dim operators are capable of masking the true alignment limit in a $2 \mathrm{HDM}$, by modifying various decay channels of the SM-like Higgs boson [32]. In the present paper, we have investigated the role of such 6-dim terms while extracting the LHC constraints on the masses of the new scalars in a 2HDM. We consider different mass spectra of these new scalars allowed from the theoretical constraints and measurements of the oblique parameters. The constraints ensuing from different searches for the heavy scalars at the LHC and possible deviations in the presence of 6-dim terms have been illustrated.

In Sec. II, we briefly review the theoretical framework of a general 2HDM. In Sec. III, we discuss the theoretical as well as phenomenological constraints on the parameter space of a $2 \mathrm{HDM}$ relevant to this work. In Sec. IV, we introduce the 6-dim terms that have been considered in this work, along with the modified couplings of the scalars. In Sec. V, we present the benchmark scenarios to illustrate the effect of such 6-dim terms on the parameter space of the $2 \mathrm{HDM}$ and eventually conclude in Sec. VI.

\section{2HDM: A REVIEW}

The two scalar doublets are defined as

$$
\varphi_{I}=\left(\begin{array}{c}
\phi_{I}^{+} \\
\frac{1}{\sqrt{2}}\left(v_{I}+\rho_{I}\right)+i \eta_{I}
\end{array}\right),
$$

with $I=1,2$. Here $\phi_{I}^{ \pm}, \rho_{I}, \eta_{I}$, and $v_{I}$ denote the charged, neutral $C P$-even and neutral $C P$-odd degrees of freedom (DOF), and the vacuum expectation value (VEV) of the $I$ th doublet, respectively.

Before spontaneous symmetry breaking (SSB), the treelevel 2HDM Lagrangian, augmented with 6-dim operators, assumes the form

$$
\mathcal{L}=\mathcal{L}_{\text {kin }}+\mathcal{L}_{\text {Yuk }}-V\left(\varphi_{1}, \varphi_{2}\right)+\mathcal{L}_{6}
$$

where

$$
\begin{aligned}
\mathcal{L}_{\mathrm{kin}}= & -\frac{1}{4} \sum_{X=G^{a}, W^{i}, B} X_{\mu \nu} X^{\mu \nu}+\sum_{I=1,2}\left|D_{\mu} \varphi_{I}\right|^{2}+\sum_{\psi=Q, L, u, d, l} \bar{\psi} i \not D \psi, \\
\mathcal{L}_{\text {Yuk }}= & \sum_{I=1,2} Y_{I}^{e} \bar{l} e \varphi_{I}+\sum_{I=1,2} Y_{I}^{d} \bar{q} d \varphi_{I}+\sum_{I=1,2} Y_{I}^{u} \bar{q} u \tilde{\varphi}_{I}, \\
V\left(\varphi_{1}, \varphi_{2}\right)= & m_{11}^{2}\left|\varphi_{1}\right|^{2}+m_{22}^{2}\left|\varphi_{2}\right|^{2}-\left(\mu^{2} \varphi_{1}^{\dagger} \varphi_{2}+\text { H.c. }\right)+\lambda_{1}\left|\varphi_{1}\right|^{4}+\lambda_{2}\left|\varphi_{2}\right|^{4}+\lambda_{3}\left|\varphi_{1}\right|^{2}\left|\varphi_{2}\right|^{2} \\
& +\lambda_{4}\left|\varphi_{1}^{\dagger} \varphi_{2}\right|^{2}+\left[\left(\frac{\lambda_{5}}{2} \varphi_{1}^{\dagger} \varphi_{2}+\lambda_{6}\left|\varphi_{1}\right|^{2}+\lambda_{7}\left|\varphi_{2}\right|^{2}\right) \varphi_{1}^{\dagger} \varphi_{2}+\text { H.c. }\right], \\
\mathcal{L}_{6}= & \sum_{i} c_{i} O_{i} / f^{2} .
\end{aligned}
$$

Here, $c_{i}$ is the Wilson coefficient of the 6-dim operator $O_{i}$, and $f$ is the scale of new physics beyond the tree-level $2 \mathrm{HDM}$. The terms proportional to $\lambda_{6,7}$ are called "hard- $Z_{2}$ violating," because they lead to a quadratically divergent amplitude for $\varphi_{1} \leftrightarrow \varphi_{2}$ transition [33] and they also lead to $C P$ violation in the scalar sector when they attain complex values [34]. But it is possible to realize the $C P$-conserving limit with nonzero values of $\lambda_{6,7}$ as well [23]. In this paper, we contain our discussion to the $C P$-conserving $2 \mathrm{HDM}$, and we take $\lambda_{6,7}=0$. The electroweak symmetry is broken by the VEVs, namely, $v_{1}$ and $v_{2}$ corresponding to the two doublets $\varphi_{1,2}$, respectively. This leads to the mixing of similar types of degrees of freedom pertaining to $\varphi_{1,2}$. In the $C P$-conserving case, the mass matrices of the neutral $C P$-even and -odd scalars and the charged scalars are diagonalized by the following field rotations:

$$
\begin{aligned}
\left(\begin{array}{c}
H \\
h
\end{array}\right) & =R(\alpha)\left(\begin{array}{l}
\rho_{1} \\
\rho_{2}
\end{array}\right), \quad\left(\begin{array}{c}
W_{L}^{ \pm} \\
H^{ \pm}
\end{array}\right)=R(\beta)\left(\begin{array}{l}
\phi_{1}^{ \pm} \\
\phi_{2}^{ \pm}
\end{array}\right), \\
\left(\begin{array}{c}
Z_{L} \\
A
\end{array}\right) & =R(\beta)\left(\begin{array}{l}
\eta_{1} \\
\eta_{2}
\end{array}\right) .
\end{aligned}
$$

Here,

$$
R(\theta)=\left[\begin{array}{cc}
\cos \theta & \sin \theta \\
-\sin \theta & \cos \theta
\end{array}\right]
$$

$h$ and $H$ are the neutral $C P$-even physical DOF, whereas $A$ and $H^{ \pm}$are the neutral $C P$-odd and charged DOF, respectively. As can be seen from Eq. (2.4), $\beta$ is the mixing angle of the charged and $C P$-odd sectors, and it is given by $\beta=\tan ^{-1}\left(v_{2} / v_{1}\right)$. $\alpha$ is the mixing angle of the $C P$-even neutral scalars and can be expressed as

$$
\alpha=\sin ^{-1}\left[\frac{\mathcal{M}_{\rho 12}^{2}}{\sqrt{\left(\mathcal{M}_{\rho 12}^{2}\right)^{2}+\left(\mathcal{M}_{\rho 11}^{2}-m_{h}^{2}\right)^{2}}}\right],
$$

with $\mathcal{M}_{\rho}^{2}$ being the mass-squared matrix in the neutral $C P$-even sector. In this paper, we assume $h$ to be the SM-like Higgs with a mass of $m_{h} \sim 125.09 \mathrm{GeV}$ and $m_{H}>m_{h}$. The case of an additional $C P$-even scalar in a 2HDM with a mass lower than the SM-like Higgs has 
been explored in the literature as well [20]. It was shown that the tree-level Higgs-mediated flavor-changing neutral currents appear in models where more than one scalar doublet gives mass to the same kind of SM fermions $[35,36]$. Such a situation can be avoided under the framework of various discrete symmetries, for example, a $Z_{2}$ symmetry $[35,36]$. There are four possible ways in which such a $Z_{2}$ charge assignment of the SM fermions can be done, namely, the type-I $\left(Y_{1}^{u}=Y_{1}^{d}=Y_{1}^{e}=0\right)$, type-II $\left(Y_{1}^{u}=Y_{2}^{d}=Y_{2}^{e}=0\right)$, type-III $\left(Y_{1}^{u}=Y_{2}^{d}=Y_{1}^{e}=0\right)$, and
type-IV $\left(Y_{1}^{u}=Y_{1}^{d}=Y_{2}^{e}=0\right)$ cases. The type-II scenario is also dubbed as the minimal supersymmetric standard model-like case due to similarity in the Yukawa sectors. Types III and IV are sometimes also referred to as flipped and lepton-specific scenarios, respectively. Because of the rotation in the scalar sector following Eq. (2.4), the couplings of the SM gauge bosons and fermions to the SM-like Higgs boson are rescaled compared to the corresponding SM values. After SSB, the Yukawa sector of the $2 \mathrm{HDM}$ can be written as

$$
\begin{aligned}
-\mathcal{L}_{\mathrm{Yuk}}= & \frac{1}{\sqrt{2}}\left(\kappa_{D} s_{\beta-\alpha}+\rho_{D} c_{\beta-\alpha}\right) \bar{D} D h+\frac{1}{\sqrt{2}}\left(\kappa_{D} c_{\beta-\alpha}-\rho_{D} s_{\beta-\alpha}\right) \bar{D} D H+\frac{1}{\sqrt{2}}\left(\kappa_{U} s_{\beta-\alpha}+\rho_{U} c_{\beta-\alpha}\right) \bar{U} U h \\
& +\frac{1}{\sqrt{2}}\left(\kappa_{U} c_{\beta-\alpha}-\rho_{U} s_{\beta-\alpha}\right) \bar{U} U H+\frac{1}{\sqrt{2}}\left(\kappa_{L} s_{\beta-\alpha}-\rho_{L} c_{\beta-\alpha}\right) \bar{L} L h+\frac{1}{\sqrt{2}}\left(\kappa_{L} c_{\beta-\alpha}-\rho_{L} s_{\beta-\alpha}\right) \bar{L} L H \\
& -\frac{i}{\sqrt{2}} \bar{U} \gamma_{5} \rho_{U} U A+\frac{i}{\sqrt{2}} \bar{D} \gamma_{5} \rho_{D} D A+\frac{i}{\sqrt{2}} \bar{L} \gamma_{5} \rho_{L} L A+\left(\bar{U}\left(V_{\mathrm{CKM}} \rho_{D} P_{R}-\rho_{U} V_{\mathrm{CKM}} P_{L}\right) D H^{+}\right. \\
& \left.+\bar{\nu} \rho_{L} P_{R} L H^{+}+\text {H.c. }\right),
\end{aligned}
$$

with $\kappa_{f}=\sqrt{2} M_{f} / v$ for $f=U, D, L$ and $\rho_{U, D, L}$ are defined in Table I. $U, D, L$, and $\nu$ represent the up-type and down-type quarks, charged leptons, and neutrinos in their mass bases, respectively. The generation indices of the fermionic fields have been suppressed in Eq. (2.7). As mentioned earlier, the measurement of the signal strengths of the SM-like Higgs at the LHC demands that the properties of one of the neutral $C P$-even neutral scalars, here $h$, should closely resemble that of the SM Higgs. As Eq. (2.7) indicates, this is satisfied at the vicinity of the so-called "alignment limit," i.e., $\cos (\beta-\alpha) \rightarrow 0$. Thus, the current measurements of Higgs signal strengths have pushed the $2 \mathrm{HDM}$ s close to the alignment limit $[19,20$, 37,38]. The measurements of the Higgs signal strengths dictate that for type-II $2 \mathrm{HDM}$, at $\tan \beta \sim 1$, the constraint on $\cos (\beta-\alpha)$ is given by $-0.05 \lesssim \cos (\beta-\alpha) \lesssim 0.15$ at $95 \%$ C.L. The allowed region becomes even smaller for higher values of $\tan \beta$. The situation for types III and IV are quite similar to that of a type-II 2HDM. This constraint is comparably relaxed in a type-I $2 \mathrm{HDM}$, where the allowed range is $|\cos (\beta-\alpha)| \lesssim 0.4$. As we are working under the assumption of a $C P$-even vacuum of the $2 \mathrm{HDM}$ potential, vertices like $A W W$ and $A Z Z$ are not present at the tree level. Among the tree-level scalar-gauge couplings which are important for the cascade decays of the new scalars, $A Z h$

TABLE I. Coupling multipliers for Yukawa interactions in 2HDM

\begin{tabular}{lcrrr}
\hline \hline & Type I & \multicolumn{1}{c}{ Type II } & Type III & Type IV \\
\hline$\rho_{D}$ & $\kappa_{D} \cot \beta$ & $-\kappa_{D} \tan \beta$ & $-\kappa_{D} \tan \beta$ & $\kappa_{D} \cot \beta$ \\
$\rho_{U}$ & $\kappa_{U} \cot \beta$ & $\kappa_{U} \cot \beta$ & $\kappa_{U} \cot \beta$ & $\kappa_{U} \cot \beta$ \\
$\rho_{L}$ & $\kappa_{L} \cot \beta$ & $-\kappa_{L} \tan \beta$ & $\kappa_{L} \cot \beta$ & $-\kappa_{L} \tan \beta$ \\
\hline \hline
\end{tabular}

and $H^{ \pm} h W^{\mp}$ are proportional to $\cos (\beta-\alpha)$, whereas $A Z H$ and $H^{ \pm} H W^{\mp}$ are proportional to $\sin (\beta-\alpha)$. It is possible to realize an exact alignment in the multi-Higgs-doublet models in the framework of certain additional symmetries of the 2HDM potential [39-43].

It is evident from Eqs. (2.7) that, when $\tan \beta \gtrsim 1$, the $h b b$ coupling multiplier deviates significantly from unity in a type-II 2HDM. For such values of $\tan \beta$, the branching ratio $\operatorname{Br}(h \rightarrow b \bar{b})$ as well as the production of $h$ in both $g g$ and $b \bar{b}$ fusion substantially increases. It is mainly due to the measurement of the processes like $g g \rightarrow \gamma \gamma, b \bar{b}, V V^{*}$ and $V h \rightarrow b \bar{b}$, that the parameter space of a type-II 2HDM in the $\cos (\beta-\alpha)-\tan \beta$ plane is quite strongly constrained. The impact of the measurement of the Higgs signal strengths in each individual search channel on the $\cos (\beta-\alpha)-\tan \beta$ plane has been discussed in Ref. [44]. It should be mentioned that the coupling multipliers of the SM-like Higgs also become close to unity when $\sin (\beta+\alpha)=1$, i.e., at the so-called "wrong-sign Yukawa" limit [45] for a type-II, -III, and -IV 2HDM. Though, with better measurement of the processes like $V h \rightarrow b \bar{b}, h \rightarrow \gamma \gamma, \Upsilon_{\gamma}[46,47]$, the fate of the wrong-sign Yukawa region will be decided in the near future.

It is also clear from Eq. (2.7) that, though the coupling multipliers of the SM-like Higgs become unity at the alignment limit, the couplings of the exotic Higgses with SM fermions can be nonzero. The $H V V$ coupling becomes identically zero at the alignment limit, protecting the alignment limit against measurements like $g g \rightarrow H \rightarrow W W, Z Z$. Though the couplings of $H$ and $A$ with SM fermions do not vanish at $\cos (\beta-\alpha)=0$. Thus, it is possible to constrain the parameter space of the type-II $2 \mathrm{HDM}$ even at the alignment limit from the nonobservation of the heavier scalars [23] in processes like $g g / b \bar{b} \rightarrow H / A \rightarrow \tau \bar{\tau}, \gamma \gamma$, 
$g g \rightarrow H \rightarrow h h$, etc. Both ATLAS and CMS are involved in numerous dedicated searches of these kinds, for instance, Ref. [48-52], resulting in significant constraints on the 2HDM parameter space.

Different kinds of mass spectra of the new scalars in a $2 \mathrm{HDM}$ can lead to quite a rich phenomenology at the LHC. In addition to the regular search channels consisting of a pair of SM particles as mentioned earlier, exotic states decaying into one another can also provide strong constraints on 2HDM parameter space. At this point, we define $m_{A}=$ $m_{H}=m_{H^{ \pm}}$as the "degenerate" case and the case when any of the three exotic scalars is more massive than the other two as the "hierarchical" case. The constraints from the decay of the new scalars into SM particles are significantly relaxed in the hierarchical scenario compared to the degenerate case [38]. But for the hierarchical spectrum of new scalars, the channels like $H(A) \rightarrow Z A(H)$ dominate the total decay width of such states, leading to new bounds on the parameter space which are not applicable for the degenerate case. A hierarchical spectrum such as $m_{A}>m_{H} \sim m_{H^{ \pm}} \sim v$ can lead to a first-order electroweak phase transition providing an explanation for the matter-antimatter asymmetry, with $A \rightarrow Z H$ being its smoking gun signature at the LHC $[53,54]$. In general, the importance of Higgs cascade decays as the possible probes of an extended scalar sector have been discussed in the literature [55-60], and $A \rightarrow Z H$ decay is dubbed as a "golden channel" in this context [61].

\section{CONSTRAINTS ON 2HDM PARAMETER SPACE}

We work with the $2 \mathrm{HDM}$ parameters in the physical basis which consists of $\left\{m_{h}, m_{H}, m_{A}, m_{H^{ \pm}}, \tan \beta, \cos (\beta-\alpha)\right.$, $\left.m_{12}^{2}, \lambda_{6}, \lambda_{7}, v\right\}$. Along with $m_{h}=125.09 \mathrm{GeV}, v=246 \mathrm{GeV}$, and $\lambda_{6,7}=0$, we are left with six free parameters. The conversion between the generic and physical basis can be found in, for instance, Refs. [16,59]. The theoretical constraints are discussed below.

(i) Vacuum stability.-The stability of the electroweak (EW) level is ensured if [16]

$$
\begin{aligned}
& \lambda_{1}>0, \quad \lambda_{2}>0, \\
& \lambda_{3}>-\sqrt{\lambda_{1} \lambda_{2}}, \quad \lambda_{3}+\lambda_{4}-\left|\lambda_{5}\right|>-\sqrt{\lambda_{1} \lambda_{2}} .
\end{aligned}
$$

It can be shown that, at the alignment limit, the first two conditions are satisfied if $m_{12}^{2}=m_{H}^{2} s_{\beta} c_{\beta}$. Along with that, the last two criteria are satisfied if $m_{h}^{2}+$ $m_{H^{ \pm}}^{2}-m_{H}^{2}>0$ and $m_{h}^{2}+m_{A}^{2}-m_{H}^{2}>0$, respectively. This means that for degenerate masses of the new scalars the last two criteria are automatically satisfied if the first two are satisfied. For the hierarchical mass spectrum, the mass of the exotic scalars cannot be arbitrarily different.

(ii) Perturbativity.-The perturbativity of the quartic couplings is satisfied if $\left|\lambda_{i}\right| \lesssim 4 \pi$. At the alignment limit, this implies that, for $t_{\beta} \gtrsim 1,\left|m_{12}^{2}-m_{H}^{2} s_{\beta} c_{\beta}\right| \lesssim v^{2}$. (iii) Unitarity.-Tree-level unitarity of the $S$ matrix requires the eigenvalues of the $2 \rightarrow 2$ scattering matrix to be less than $8 \pi$. At the alignment limit for $m_{12}^{2} \sim m_{H}^{2} s_{\beta} c_{\beta}$, this implies that the differences between the masses of the new scalars have to be $\lesssim v$.

(iv) Oblique parameters. - The new scalars in 2HDM contribute to the oblique parameters through their couplings to the massive gauge bosons [62-67]. At the alignment limit, such contributions to the $T$ parameter assume the form

$$
\begin{aligned}
\Delta T= & \frac{g^{2}}{64 \pi^{2} m_{W}^{2}}\left(F\left(m_{H^{ \pm}}^{2}, m_{A}^{2}\right)+F\left(m_{H^{ \pm}}^{2}, m_{H}^{2}\right)\right. \\
& \left.-F\left(m_{A}^{2}, m_{H}^{2}\right)\right),
\end{aligned}
$$

with

$$
\begin{aligned}
F(a, b) & =\frac{a+b}{2}-\frac{a b}{a-b} \ln \left(\frac{a}{b}\right) \quad(a \neq b) \\
& =0 \quad(a=b) .
\end{aligned}
$$

As Eq. (3.2) suggests, this anomalous contribution to the $T$ parameter vanishes at the limit $m_{H^{ \pm}}=m_{A}$ or $m_{H^{ \pm}}=m_{H}$. However, substantially away from the alignment limit, this does not hold for the entire range of $m_{A}$. As mentioned earlier, the measurements of the Higgs signal strengths imply that the maximum values of $\cos (\beta-\alpha)$ can be attained in a type-I 2HDM, $|\cos (\beta-\alpha)| \lesssim 0.4$. For the remaining three types of Yukawa structure, $|\cos (\beta-\alpha)| \lesssim 0.1$. We have checked that the limits $m_{H}=m_{H^{ \pm}}$and $m_{A}=m_{H^{ \pm}}$ensure that the contribution to the $T$ parameter remains in the experimentally allowed range at $95 \%$ C.L. even when we consider small deviations from the alignment limit, $\cos (\beta-\alpha) \lesssim$ 0.1 . For $\cos (\beta-\alpha) \sim 0.4$, even for $m_{H}=m_{H^{ \pm}}=$ $300 \mathrm{GeV}, m_{A} \gtrsim 480 \mathrm{GeV}$ is ruled out from the measurement of the $T$ parameter.

LEP searches put a constraint on the mass of the charged scalar as $m_{H^{ \pm}} \gtrsim 72 \mathrm{GeV}(80 \mathrm{GeV}$ ) for a type-I (II) $2 \mathrm{HDM}$ [68]. Also, the searches for $Z \rightarrow A H \rightarrow \tau \bar{\tau} \tau \bar{\tau}$ lead to the constraint $m_{H}+m_{A} \gtrsim 208 \mathrm{GeV}$ [69]. The charged scalar mediates flavor-violating processes such as $B_{d} \rightarrow X_{s} \gamma$, $B_{s} \rightarrow \mu^{+} \mu^{-}, B_{d}^{+} \rightarrow \tau^{+} \nu$, etc., which in turn lead to constraints on $m_{H}^{ \pm}$[70-72]. The measurement of the width of $B_{d} \rightarrow X_{s} \gamma$ leads to the most stringent constraint on the charged scalar mass for a type-II $2 \mathrm{HDM}, m_{H^{+}} \gtrsim 580 \mathrm{GeV}$, almost independent of the value of $\tan \beta$ [72]. For a type-I $2 \mathrm{HDM}$, the constraint from meson decays is comparatively less stringent and depends rather strongly on $\tan \beta$. For $\tan \beta \sim 1.5$, the constraint is $m_{H^{+}} \gtrsim 200 \mathrm{GeV}$ [72]. Based on the similarity in couplings of the scalars to the quarks, the constraints on a charged scalar mass for a type-I and -II 2HDM can also be used for type-IV and -III cases, 
respectively, though we do not consider this as a hard bound for our purpose as it can be ameliorated in several extensions of 2HDM [73]. Moreover, the mass differences of the three exotic scalars are constrained from the measurement of the oblique parameters [62]. For $\cos (\beta-\alpha)=0$, the contributions of the new scalars to the $S$ and $T$ parameters do not depend upon $m_{12}^{2}$ or $\tan \beta$. For a fixed value of $m_{H}$, the constraints from precision tests are satisfied only if $m_{H} \sim m_{A}$ or $m_{H} \sim m_{H^{ \pm}}$. As mentioned earlier, the measurements of the signal strengths of the SM-like Higgs boson constrain the value of $\cos (\beta-\alpha)$ to be close to zero, especially for a type-II, -III, and -IV 2HDM. Thus, while working with the hierarchical spectrum of the new scalars, we take $\cos (\beta-\alpha)$ to be close to zero. But for the degenerate spectrum of exotic scalars, we consider a possible large deviation from the alignment limit while we present the excluded region on the $\cos (\beta-\alpha)-\tan \beta$ plane. In this paper, we have considered the constraints due to the nonobservation of the processes: $g g \rightarrow H \rightarrow Z Z$ [48], $g g \rightarrow H \rightarrow h h$ [49], $g g \rightarrow H(A) \rightarrow$ $A(H / h) Z$ [50], and $g g / b \bar{b} \rightarrow H / A \rightarrow \tau \bar{\tau}$ [51,52].

\section{COUPLINGS OF THE HEAVIER SCALARS IN 2HDMEFT}

We contain our discussion only to the bosonic operators of 2HDMEFT for simplicity. The phenomenology of the fermionic dim- 6 terms will be reported elsewhere. As discussed in Ref. [32], the measurement of EW oblique parameters, triple gauge boson vertices, and Higgs signal strengths constrains the bosonic operators other than type $\varphi^{4} D^{2}$ at $\mathcal{O}\left(10^{-3}\right)$. Moreover, some of the $\varphi^{4} D^{2}$ type of operators violate the $T$ parameter at the tree level, and the corresponding Wilson coefficients are rather small. Thus, we have considered only the operators of type $\varphi^{4} D^{2}$ which do not contribute to the $T$ parameter in the tree level [26]:

$$
\begin{aligned}
O_{H 1} & =\left(\partial_{\mu}\left|\varphi_{1}\right|^{2}\right)^{2}, \\
O_{H 2} & =\left(\partial_{\mu}\left|\varphi_{2}\right|^{2}\right)^{2}, \\
O_{H 12} & =\left(\partial_{\mu}\left(\varphi_{1}^{\dagger} \varphi_{2}+\text { H.c. }\right)\right)^{2}, \\
O_{H 1 H 2} & =\partial_{\mu}\left|\varphi_{1}\right|^{2} \partial^{\mu}\left|\varphi_{2}\right|^{2}, \\
O_{H 1 H 12} & =\partial_{\mu}\left|\varphi_{1}\right|^{2} \partial^{\mu}\left(\varphi_{1}^{\dagger} \varphi_{2}+\text { H.c. }\right), \\
O_{H 2 H 12} & =\partial_{\mu}\left|\varphi_{2}\right|^{2} \partial^{\mu}\left(\varphi_{1}^{\dagger} \varphi_{2}+\text { H.c. }\right) .
\end{aligned}
$$

In the presence of such operators, the nondiagonal kinetic terms arise after SSB [26]. In order to get rid of such terms, one needs to rescale the neutral $C P$-even DOF, i.e., $\rho_{1}$ and $\rho_{2}$. This implies that the physical neutral $C P$-even scalars in the presence of these operators are rescaled compared to the tree-level 2HDM:

$$
\begin{gathered}
h \rightarrow\left(1-x_{1}\right) h+y H, \\
H \rightarrow\left(1-x_{2}\right) H+y h .
\end{gathered}
$$

$x_{1}, x_{2}$, and $y$ can be written in terms of the Wilson coefficients of the operators appearing in Eq. (4.1) and the scale of new physics beyond the 2HDM. The analytical forms of $x_{1}, x_{2}$, and $y$ can be found in Appendix A. In our convention, the Wilson coefficient of an operator $O_{i}$ is given as $c_{i}$, which includes the symmetry factors. Equation (4.2) dictates that any coupling involving at least one $h$ or $H$ field is modified compared to the 2HDM at the tree level. For example,

$$
\begin{gathered}
\kappa_{h f f}^{\prime}=\left(1-x_{1}\right) \kappa_{h f f}+y \kappa_{H f f}, \\
\kappa_{H f f}^{\prime}=\left(1-x_{2}\right) \kappa_{H f f}+y \kappa_{h f f}, \\
\kappa_{h V V}^{\prime}=\left(1-x_{1}\right) \sin (\beta-\alpha)+y \cos (\beta-\alpha), \\
\kappa_{H V V}^{\prime}=\left(1-x_{2}\right) \cos (\beta-\alpha)+y \sin (\beta-\alpha), \\
\kappa_{A Z h}^{\prime}=\left(1-x_{1}\right) \kappa_{A Z h}+y \kappa_{A Z H}, \\
\kappa_{A Z H}^{\prime}=\left(1-x_{2}\right) \kappa_{A Z H}+y \kappa_{A Z h} .
\end{gathered}
$$

Equations (4.4) and (4.6) affect decay processes like $H \rightarrow \tau \bar{\tau}$ and $H \rightarrow Z Z$, which are particularly important in the degenerate case. Similarly, the decay widths $H(A) \rightarrow$ $Z A(H)$, which become relevant in the hierarchical scenarios, are changed according to Eq. (4.8). What is more interesting, processes, which apparently look unaffected by the rescaling of fields, such as $g g \rightarrow A \rightarrow \tau \bar{\tau}$, are also changed. It is due to the fact that away from the alignment limit, for a large range of values of $m_{A}$ and $\tan \beta, \operatorname{Br}(A \rightarrow$ $Z h)$ is quite significant. The change in $\operatorname{Br}(A \rightarrow Z h)$ according to Eq. (4.7) in turn modifies $\operatorname{Br}(A \rightarrow \tau \bar{\tau})$. Even the change in $h \rightarrow f \bar{f}$ can be important for bounds on the heavier scalars. For example, the search for $A \rightarrow Z h$ assumes that the Higgs in the final state further decays into a pair of $b$-tagged jets [50]. Moreover, the coupling multipliers of the SM-like Higgs also modify upon the inclusion of dim-6 operators compared to the tree-level 2HDM according to Eqs. (4.3) and (4.5). Thus, the allowed parameter space changes in the $\cos (\beta-\alpha)-\tan \beta$ plane [32].

Many of the sum rules involving various gauge couplings, which hold in the 2HDM at the tree level, are no longer valid in the presence of 6-dim operators [4]. These sum rules can play an important role in deciphering new physics beyond the 2HDM. For instance, in the 2HDM at the tree level, the sum rule $\kappa_{h V V}^{2}+\kappa_{H V V}^{2}=1$ holds true, but in the presence of the dim- 6 terms mentioned in Eq. (4.1), $\kappa_{h V V}^{2}+\kappa_{H V V}^{2}=1-2\left(x_{1} s_{\beta-\alpha}^{2}+x_{2} c_{\beta-\alpha}^{2}+2 y c_{\beta-\alpha} s_{\beta-\alpha}\right)$. If another $C P$-even neutral scalar $H$ is discovered after $h(125)$, the measurement of its decay width and $\operatorname{Br}(H \rightarrow W W)$ will facilitate the verification of such a sum rule. A deviation from $\kappa_{h V V}^{2}+\kappa_{H V V}^{2}=1$ will point to a departure from the $C P$-conserving 2HDM. If $\kappa_{h V V}^{2}+\kappa_{H V V}^{2}<1$, then it may 
indicate towards a $C P$-violating $2 \mathrm{HDM}$ or $C P$-conserving $N$-Higgs-doublet-model $(N>2)$. But such an interpretation does not hold if there are more than one neutral BSM scalars with degenerate masses or masses the same as the SM-like Higgs. Anyway, even the dim-6 operators in 2HDMEFT can lead to $\kappa_{h V V}^{2}+\kappa_{H V V}^{2}<1$. On contrary, neither a $\mathrm{CP}$-violating $2 \mathrm{HDM}$ nor $\mathrm{N}$-Higgs-doublet-model can lead to $\kappa_{h V V}^{2}+\kappa_{H V V}^{2}>1$, though such a scenario can be interpreted in terms of the dim- 6 terms of 2HDMEFT. At the $C P$-conserving limit with $\lambda_{6,7} \neq 0$, the similar argument for a tree-level $2 \mathrm{HDM}$ is valid in this context, whereas, in general, $\lambda_{6,7} \neq 0$ will follow the argument for a $C P$-violating $2 \mathrm{HDM}$.

\section{BENCHMARK SCENARIOS}

Following the discussions in Sec. III, in the context of oblique parameters, for the hierarchical mass spectrum, we consider either $m_{A}=m_{H^{ \pm}}$or $m_{H}=m_{H^{ \pm}}$. The limit $m_{A}=m_{H}$ is highly constrained from the measurement of $S$, $T$ parameters, and the decays of $H$ and $A$ into each other are kinematically forbidden.

So the mass spectra under scrutiny for the hierarchical case are [59]
C1: $m_{A}=m_{H^{ \pm}}>m_{H}$,
C2: $m_{A}>m_{H}=m_{H^{ \pm}}$,
C3: $m_{A}=m_{H^{ \pm}}<m_{H}$,
C4: $m_{A}<m_{H}=m_{H^{ \pm}}$.

For the hierarchical case, we have studied the bounds on the $m_{A}=m_{H}$ plane for $\tan \beta=1.5$ and $\cos (\beta-\alpha)=0$ with and without considering the dim- 6 terms.

On the other hand, for the degenerate mass spectrum $\left(m_{H}=m_{A}=m_{H^{ \pm}}\right)$, new scalars can no longer decay into each other, thus making the SM decay channels of these scalars $H \rightarrow Z Z, \tau \bar{\tau}, b \bar{b}, A \rightarrow Z h, \tau \bar{\tau}$ more important. We have studied the change in the constraints due to the inclusion of dim- 6 operators on the $\cos (\beta-\alpha)-m_{A}$ plane. We define the benchmark for the degenerate case as

$$
\text { C5: } m_{H}=m_{A}=m_{H^{ \pm}}, \quad \tan \beta=1.5 .
$$

As mentioned earlier, $\lambda_{6,7}=0$ for both degenerate and hierarchical cases. Also, the quadratic mass parameter is taken to be $m_{12}^{2}=m_{H}^{2} s_{\beta} c_{\beta}$ following the discussions on the theoretical constraints in Sec. III. For both the hierarchical and degenerate cases, the bounds on the 2HDM at the tree level are compared with a specific case of 2HDMEFT with the following values of the Wilson coefficients and the new physics scale [32]:

$$
\begin{aligned}
& \text { BP1: } c_{H 1}=-1, \quad c_{H 2}=1.5, \\
& c_{H 12}=c_{H 1 H 2}=c_{H 1 H 12}=c_{H 2 H 12}=0, \\
& f=1 \mathrm{TeV} .
\end{aligned}
$$

The theoretical constraints such as perturbativity and stability do not change upon the inclusion of bosonic operators considered in this paper, as these operators do not modify the 2HDM scalar potential. However, they can lead to additional contributions in the $S$ matrix for $2 \rightarrow 2$ scattering of bosonic states. Implementing these changes in 2HDMC-1.7.0 [74], we have checked that, for the 2HDMEFT benchmark scenario BP1, there are no significant modifications of the allowed parameter space for $\sqrt{\hat{s}} \sim$ few TeV while $f=1 \mathrm{TeV}$. Similar conclusions were obtained for a composite $2 \mathrm{HDM}$ based on $S O(6) / S O(4) \times S O(2)$ [75].

The branching ratios of various scalars in the $2 \mathrm{HDM}$ at the tree level, as well as in the presence of the 6-dim operators, have been calculated with 2HDMC-1.7.0 [74] after incorporating the modified couplings. The production cross section of the neutral scalars has been computed up to nextto-next-to-leading order in QCD using SusHi-1.6.1 [76]. As mentioned earlier, the constraints on the $\cos (\beta-\alpha)-\tan \beta$ plane from the measurement of signal strengths of $h(125)$ change in the presence of the dim- 6 operators. For instance, in a type-II $2 \mathrm{HDM}$ for $\tan \beta=1.5$, the allowed range of $\cos (\beta-\alpha)$ in the 2HDM is $[-0.05,0.12]$, which changes in BP1 of 2HDMEFT to $[-0.02,0.11]$ [32]. The allowed range of $\cos (\beta-\alpha)$ changes for the other types of Yukawa couplings as well, but the exact tree-level alignment limit $\cos (\beta-\alpha)=0$ is allowed in all these cases for $\tan \beta=1.5$.

The constraints on the $m_{A}-m_{H}$ plane from various exotic Higgs search channels are elucidated in Figs. 1-4 corresponding to the mass spectra $\mathbf{C 1}, \mathbf{C 2}, \mathbf{C 3}$, and $\mathbf{C 4}$, respectively. In Fig. 1, the appreciable change from the treelevel 2HDM scenario takes place only for type-I and typeIV Yukawa couplings. Moreover, it can be seen that the characteristics of the type-I and -IV 2HDM are similar in this context, as is the case for type II and type III. This pattern can be attributed to the similarity in the couplings of the new scalars with the SM quarks, following Eq. (2.7), which dictate their production cross sections at the LHC. The most significant search channel in the context of the mass spectra $\mathbf{C 1}$ is $A \rightarrow Z H(b \bar{b})$. For all four Yukawa types, the change in $\operatorname{Br}(H \rightarrow b \bar{b})$ can be substantially different in 2HDMEFT compared to a tree-level 2HDM, though $\operatorname{Br}(A \rightarrow Z H)$ does not change significantly. This happens because a key decay channel of $H$, i.e., $H \rightarrow W W$, becomes viable in BP1 of 2HDMEFT, which is absent in the tree-level 2HDM at $\cos (\beta-\alpha)=0$. For example, with $m_{A}=400 \mathrm{GeV}$ and $m_{H}=300 \mathrm{GeV}, \operatorname{Br}(H \rightarrow b \bar{b})$ becomes $\sim 0.45$ in BP1 of 2HDMEFT compared to its value $\sim 0.73$ in the tree-level $2 \mathrm{HDM}$. Such a reduction in $\operatorname{Br}(H \rightarrow b \bar{b})$ is compensated by the newly viable channel $H \rightarrow W W$. This leads to a reduced value of $A \rightarrow Z H(b \bar{b})$ and, eventually, to a more relaxed constraint on $m_{H}$. Compared to type-I and -IV 2HDMs, $\operatorname{Br}(H \rightarrow b \bar{b})$ is larger for type-II and -III Yukawas even in the tree-level scenario. Thus, the percentage change in $\operatorname{Br}(H \rightarrow b \bar{b})$ is much lower for types II and III compared to types I and IV. So the change in the excluded region from the nonobservation of $A \rightarrow Z H$ 


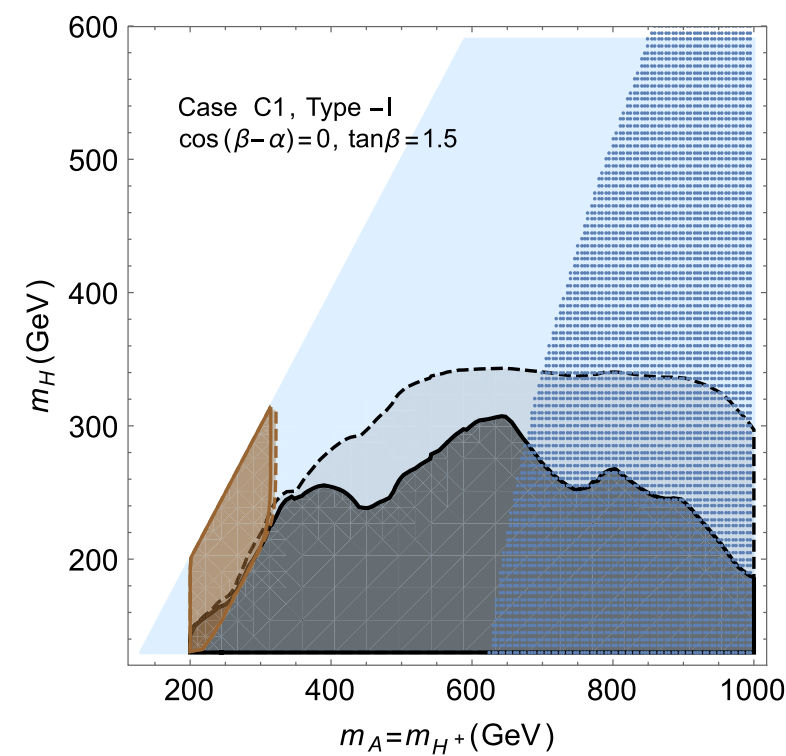

(a)

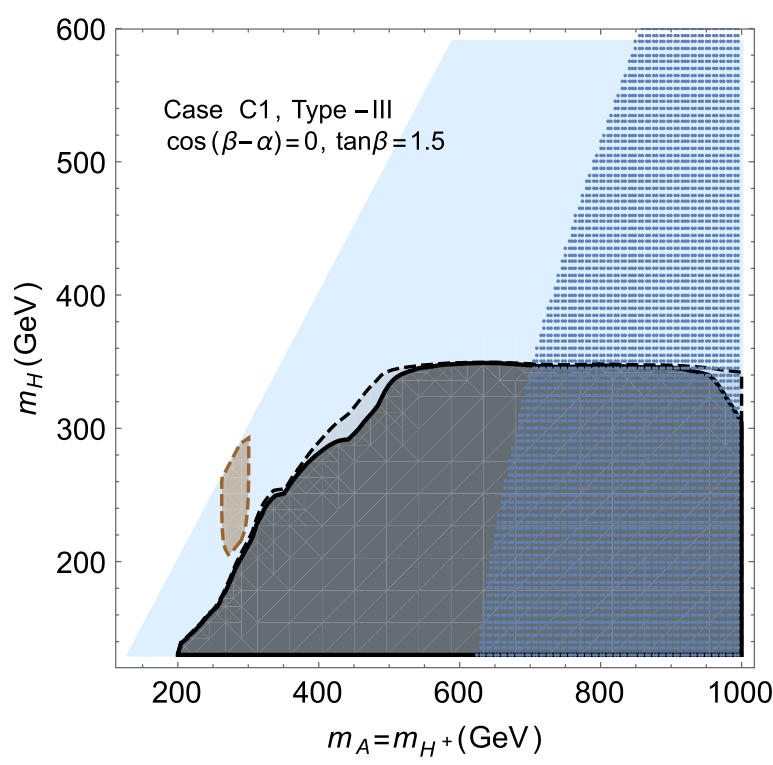

(c)

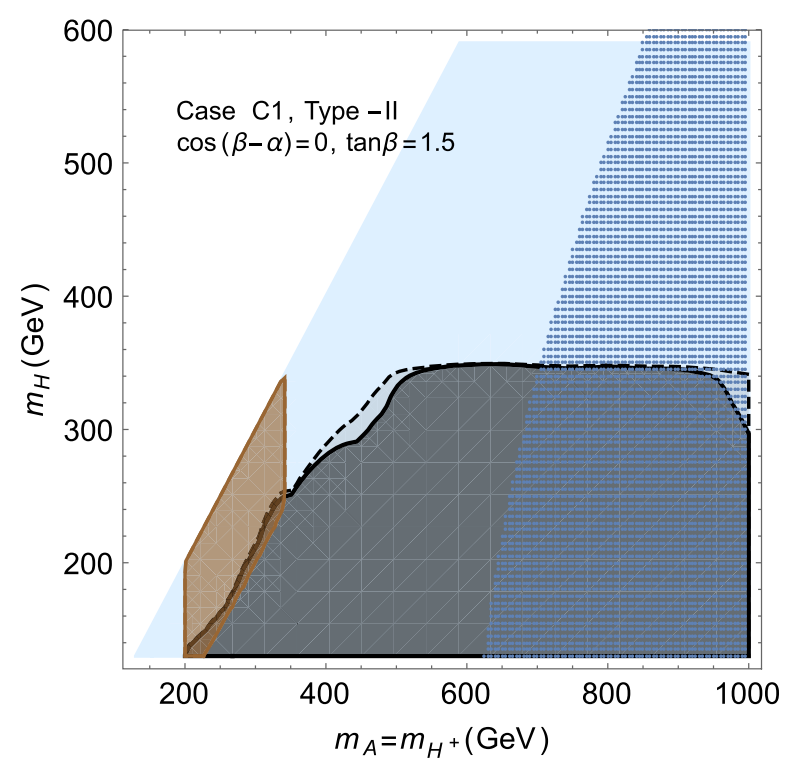

(b)

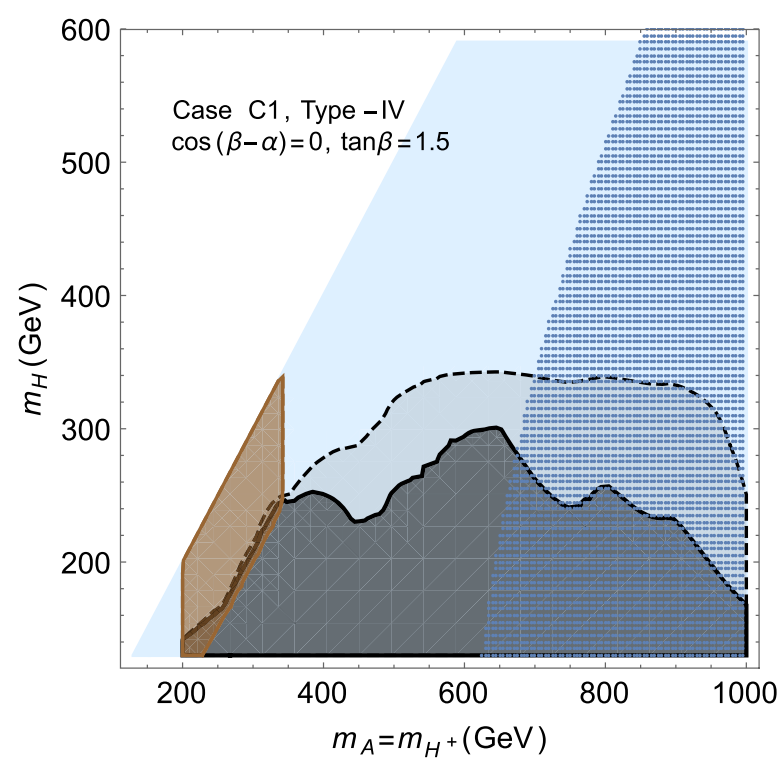

(d)

FIG. 1. The effect of the $\varphi^{4} D^{2}$ type of operators for mass spectrum C1. Only the colored regions are kinematically viable. The gray regions with the dashed and solid boundaries are ruled out from $g g \rightarrow A \rightarrow Z H(b \bar{b})$ [50] in a 2HDM and BP1 of 2HDMEFT, respectively. The brown regions with the dashed and solid boundaries are ruled out from $g g \rightarrow A \rightarrow \tau \bar{\tau}$ [51] in a 2HDM and $\mathbf{B P 1}$ of 2HDMEFT, respectively. The meshed blue region is disfavored from the theoretical constraints, viz. stability, perturbativity, and unitarity.

is larger in types I and IV compared to types II and III. $A \rightarrow \tau \bar{\tau}$ rules out a region of parameter space where the on-shell decay $A \rightarrow Z H$ is not allowed. As Eq. (2.7) suggests, $\operatorname{Br}(A \rightarrow \tau \bar{\tau})$ attains the smallest value for the type-III case among all the Yukawa types. In both types I and III, the $A \tau \bar{\tau}$ coupling is proportional to $\cot \beta$ as opposed to $\tan \beta$ in types II and IV. $\operatorname{Br}(A \rightarrow \tau \bar{\tau})$ is even smaller in a type-III 2HDM compared to a type-I $2 \mathrm{HDM}$, because $\operatorname{Br}(A \rightarrow b \bar{b})$ becomes larger in the latter case. On the inclusion of the dim- 6 operators, the region excluded from $g g \rightarrow A \rightarrow \tau \bar{\tau}$ is not significantly altered for the type-I, -II, and -IV cases.

In the context of Yukawa types, a similar pattern in the excluded region can be seen for mass spectra C2 as depicted in Fig. 2. It can also be seen that the excluded region from $A \rightarrow Z H$ is small in case $\mathbf{C 2}$ compared to $\mathbf{C 1}$. For mass spectrum $\mathbf{C 2}$, the decay channel $A \rightarrow W^{ \pm} H^{\mp}$ becomes kinematically viable and has a branching ratio almost similar to that of $A \rightarrow Z H$. However, the branching 


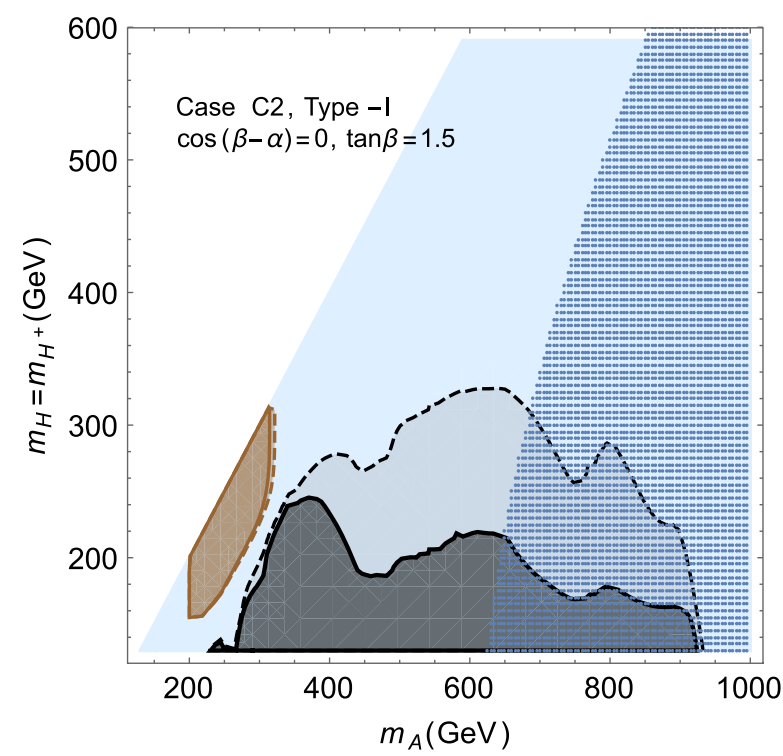

(a)

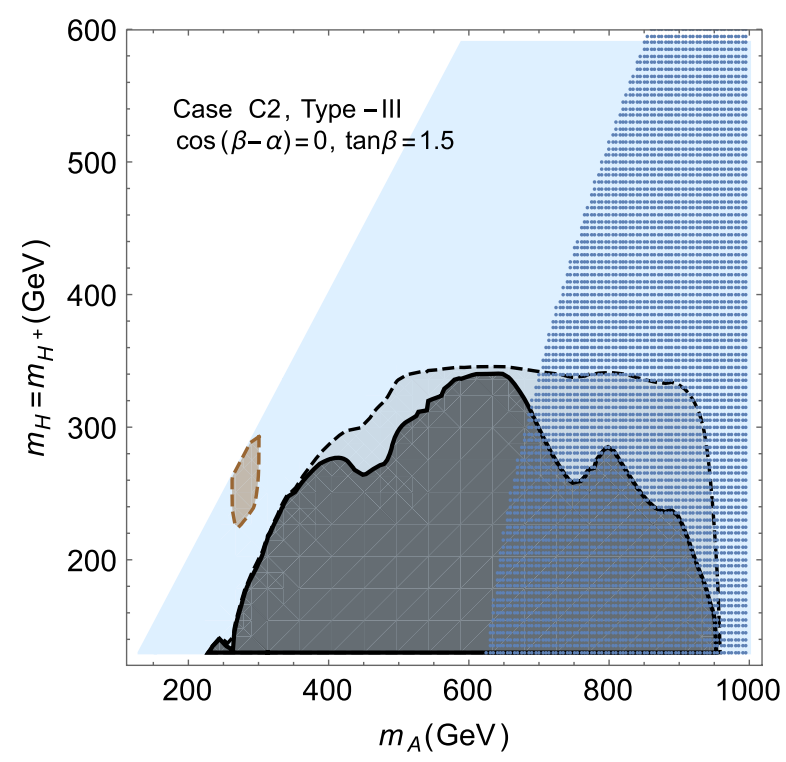

(c)

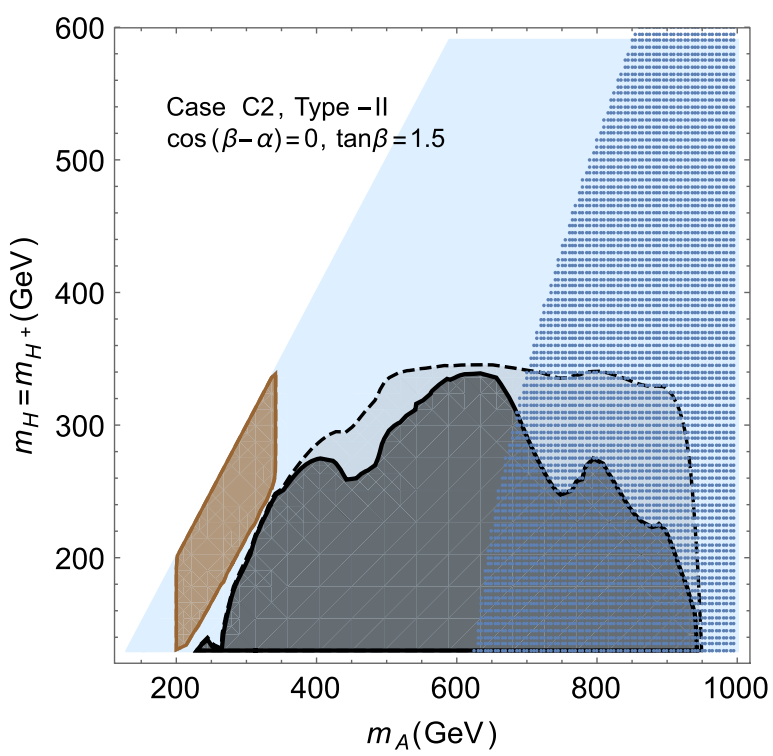

(b)

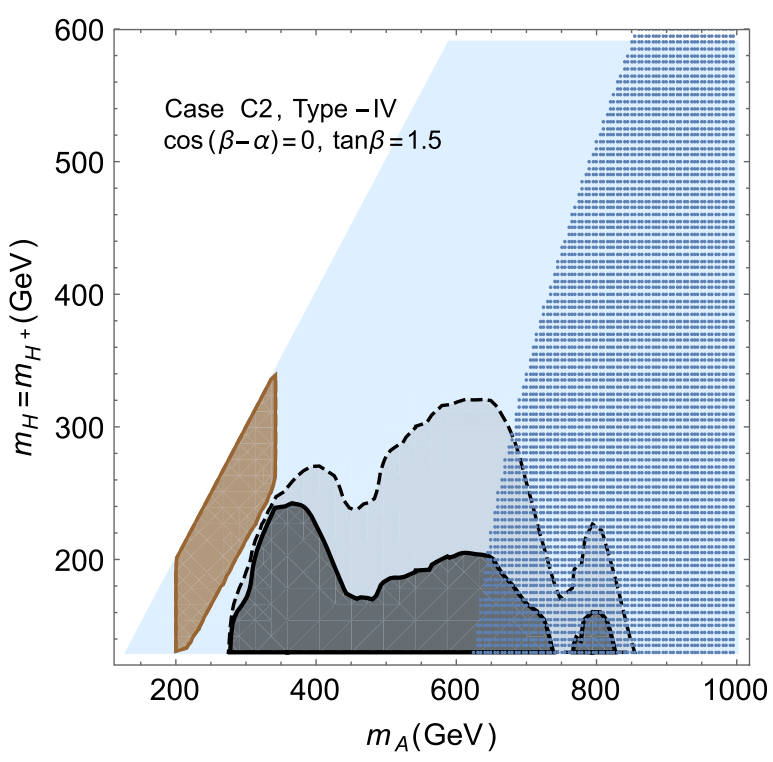

(d)

FIG. 2. The effect of the $\varphi^{4} D^{2}$ type of operators for mass spectrum C2. Color coding is the same as in Fig. 1 .

ratios of $H$ are the same in cases $\mathbf{C 1}$ and $\mathbf{C 2}$. This, along with $\left.\operatorname{Br}(A \rightarrow Z H)\right|_{\mathbf{C} 1} \gtrsim 2 \times\left.\operatorname{Br}(A \rightarrow Z H)\right|_{\mathbf{C} 2}$, dictates that the excluded region for $\mathbf{C 1}$ is larger than that in $\mathbf{C 2}$. As the branching ratios of $H$ in cases $\mathbf{C 1}$ and $\mathbf{C 2}$ are the same in both a 2HDM and 2HDMEFT, the patterns of deviation in terms of different Yukawa couplings are similar for $\mathbf{C 1}$ and $\mathbf{C 2}$. The changes in the branching ratios have been illustrated in Figs. 8 and 9 in Appendix B.

$H \rightarrow Z A(b \bar{b})$ can rule out a significant area of parameter space on the $m_{A}-m_{H}$ plane for cases $\mathbf{C 3}$ and C4. The excluded region in case $\mathbf{C} \mathbf{4}$ does not show a substantial change upon the inclusion of dim-6 terms as in $\mathbf{C 1}$ or $\mathbf{C 2}$.
This happens because $\operatorname{Br}(A \rightarrow b \bar{b})$ remains almost the same in a $2 \mathrm{HDM}$ and $2 \mathrm{HDMEFT}$. It changes only by $\lesssim 10 \%$ due to a small nonzero value of $\operatorname{Br}(A \rightarrow Z h)$ in BP1 of 2HDMEFT. As opposed to 2HDMEFT, for $\cos (\beta-\alpha)=0, A \rightarrow Z h$ is absent in the 2HDM at the tree level. So the constraints on $m_{A}$ from $H \rightarrow Z A$ can be relaxed at most by $\sim 19 \mathrm{GeV}$ for types I and IV for $\mathbf{C 4}$ around $m_{H} \sim 795 \mathrm{GeV}$, a region which is already disfavored from the criteria of stability and unitarity. It can be seen that a significant area is ruled out from $H \rightarrow W W$ for $m_{H} \lesssim 350 \mathrm{GeV}$ and $m_{H}-m_{A} \lesssim 90 \mathrm{GeV}$ if the 6-dim operators are included. $H \rightarrow W W$ is otherwise absent in 


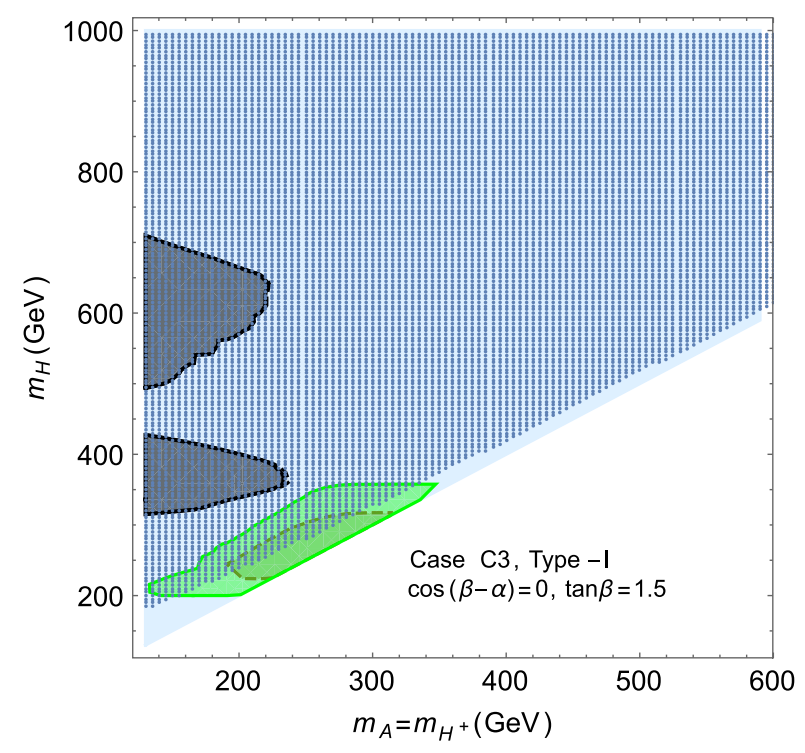

(a)

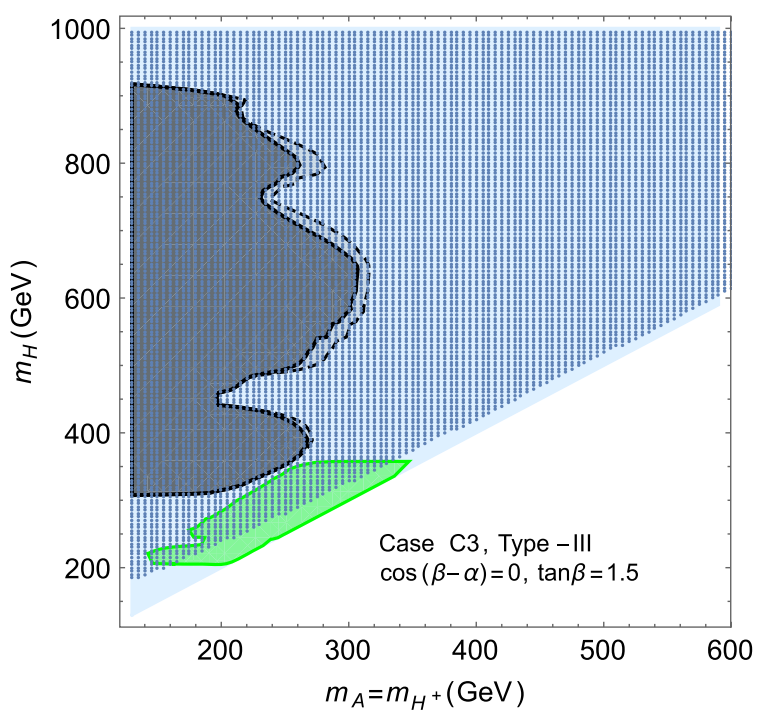

(c)

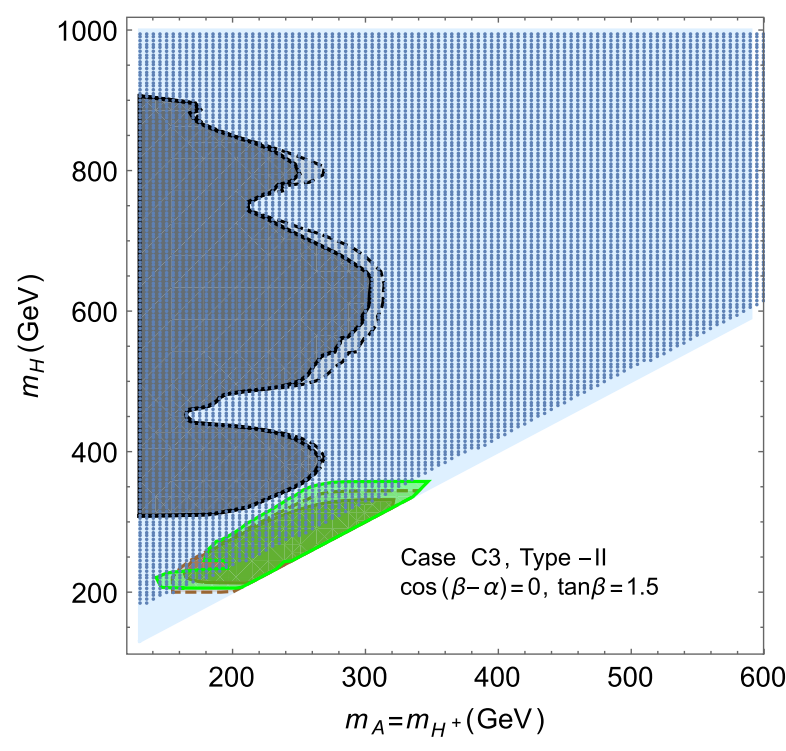

(b)

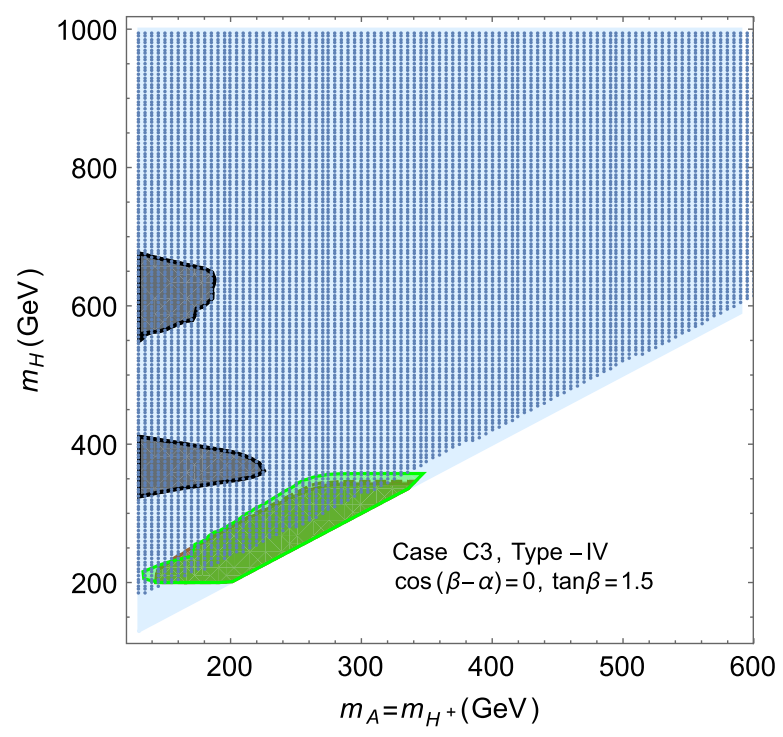

(d)

FIG. 3. The effect of the $\varphi^{4} D^{2}$ type of operators for mass spectrum C3. The gray regions with the dashed and solid boundaries are ruled out from $g g \rightarrow H \rightarrow Z A(b \bar{b})$ [50] in a 2HDM and BP1 of 2HDMEFT, respectively. The brown regions with the dashed and solid boundaries are ruled out from $g g \rightarrow H \rightarrow \tau \bar{\tau}$ [51] in a 2HDM and BP1 of 2HDMEFT, respectively. The green region is disfavored from the nonobservation of $g g \rightarrow H \rightarrow Z Z$ [48] in BP1 of 2HDMEFT, which is absent in the 2HDM at the tree level for $\cos (\beta-\alpha)=0$. The rest of the color coding is the same as in Fig. 1.

the alignment limit irrespective of the mass spectrum of the new scalars. $H \rightarrow t \bar{t}$ becomes dominant if $m_{H} \gtrsim$ $2 m_{t} \sim 350 \mathrm{GeV}$. For $m_{H} \lesssim 350 \mathrm{GeV}, \quad \operatorname{Br}(H \rightarrow Z A)$ becomes substantial if $m_{H}-m_{A} \gtrsim 90 \mathrm{GeV}$. Thus, the area disfavored by $H \rightarrow W W$ is confined to a small strip close to the $m_{H} \sim m_{A}$ line as shown in Fig. 4. A similar effect can be seen in Fig. 3 for the case C3. The appearance of such an exclusion region originates from the fact that, in the presence of the 6-dim terms, the coupling multiplier $\kappa_{H V V}$ does not vanish at $\cos (\beta-\alpha)=0$ as opposed to the tree-level 2HDM. The region ruled out from $H \rightarrow \tau \bar{\tau}$ overlaps with that for $H \rightarrow W W$ in 2HDMEFT in most of the cases. However, for type-I and -III 2HDMs, the constraint from $H \rightarrow \tau \bar{\tau}$ appears to be relaxed compared to the two other types. It can also be seen that the region excluded from $H \rightarrow Z A$ is smaller for $\mathbf{C} 3$ compared to $\mathbf{C 4}$ even in a $2 \mathrm{HDM}$ at tree level. This occurs because the decay channel $H \rightarrow W^{ \pm} H^{\mp}$ becomes kinematically viable for $\mathbf{C 3}$ and $\operatorname{Br}\left(H \rightarrow W^{ \pm} H^{\mp}\right) \sim \operatorname{Br}(H \rightarrow Z A)$. This leads to a smaller cross section in the channel $g g \rightarrow H \rightarrow Z A$ and, 


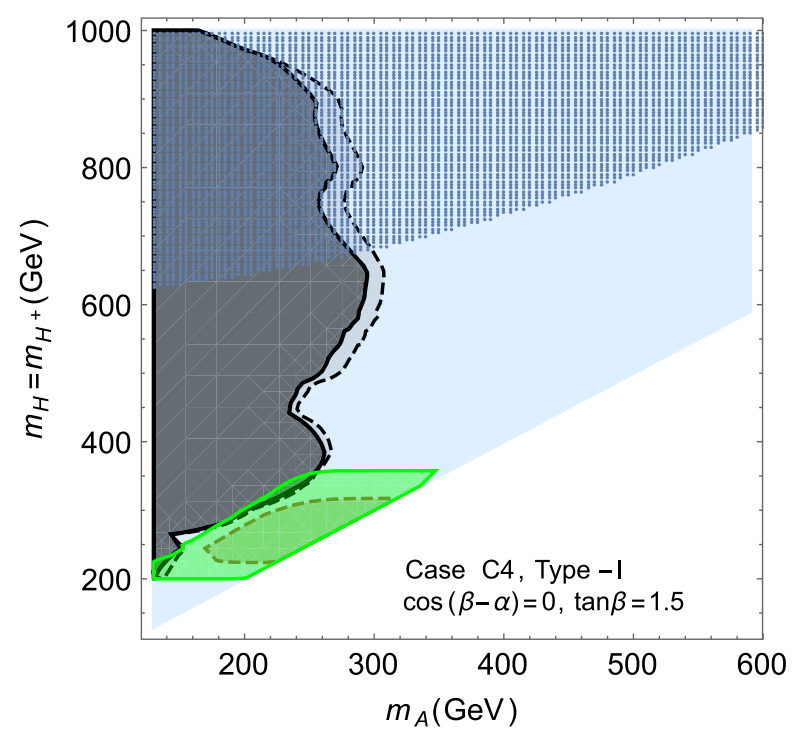

(a)

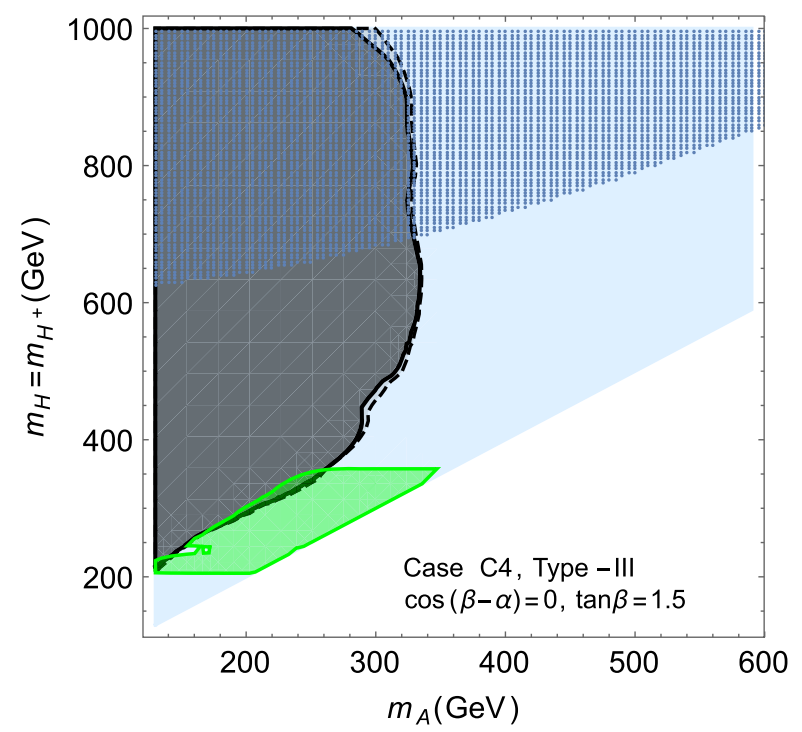

(c)

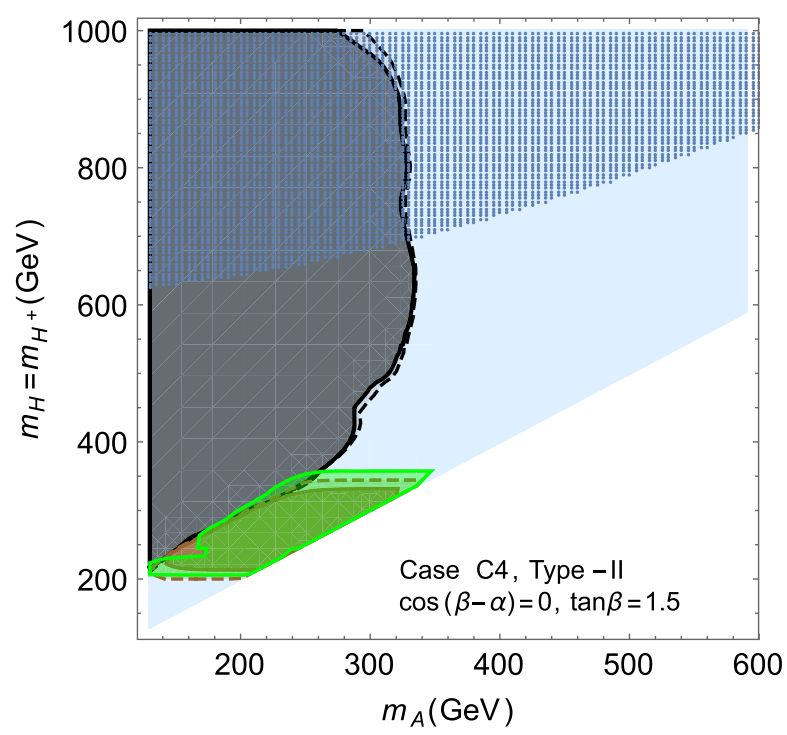

(b)

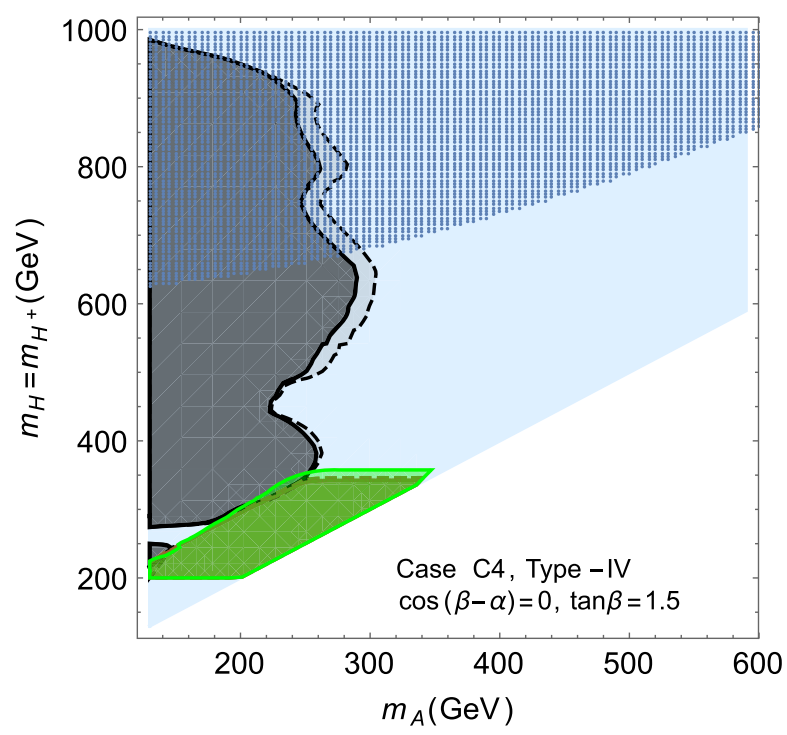

(d)

FIG. 4. The effect of the $\varphi^{4} D^{2}$ type of operators for mass spectrum C4. Color coding is the same as in Fig. 3.

hence, a relaxed constraint on $m_{A}$ for $\mathbf{C} \mathbf{3}$ compared to the case $\mathbf{C 4}$ even in a tree-level 2HDM.

Till now, we have discussed the changes in the excluded region on the $m_{A}-m_{H}$ plane at a low value of $\tan \beta$. At large $\tan \beta$, i.e., $\tan \beta \gtrsim 10$, exotic decays such as $H \rightarrow Z A, A \rightarrow Z H$ become negligible even in the hierarchical scenarios, and $g g / b \bar{b} \rightarrow H / A \rightarrow \tau \bar{\tau}$ leads to the only relevant constraint. This can be read off Eq. (2.7). In such cases, for $\cos (\beta-\alpha)=0$, the constraints on $m_{H}$ or $m_{A}$ are altered at the most by $\sim 5 \mathrm{GeV}$.

In Fig. 5(a), we have depicted the constraints on the $\cos (\beta-\alpha)-m_{A}$ plane due to the nonobservation of $H$ and $A$ in the degenerate mass scenario for a type-II 2HDM. As mentioned earlier, the exotic decay channels like
$H(A) \rightarrow A(H) Z$, etc., are absent in such a case. It can be seen from Eq. (2.7) that couplings like $H V V, A Z h$, etc., vanish at the exact alignment limit in a $2 \mathrm{HDM}$ at the tree level [23]. Thus, the nonobservation of $H \rightarrow Z Z$ or $A \rightarrow Z h$ cannot rule out $\cos (\beta-\alpha)=0$ irrespective of the value of $m_{A}$. This also implies that the discovery of a new scalar in the $V V$ or $Z h$ final states would rule out the exact alignment limit in the framework of a $C P$-conserving 2HDM. Though it is not the case if the 6-dim terms are also present, as those can lead to a nonvanishing contribution to such decay channels. It can be seen from Fig. 5(a) that, in a 2HDM augmented with the $\varphi^{4} D^{2}$ kind of operators, the bounds from $H \rightarrow Z Z, A \rightarrow \tau \bar{\tau}$, $Z h$ modify in comparison to a $2 \mathrm{HDM}$ at the tree level. $H \rightarrow Z Z$ can 


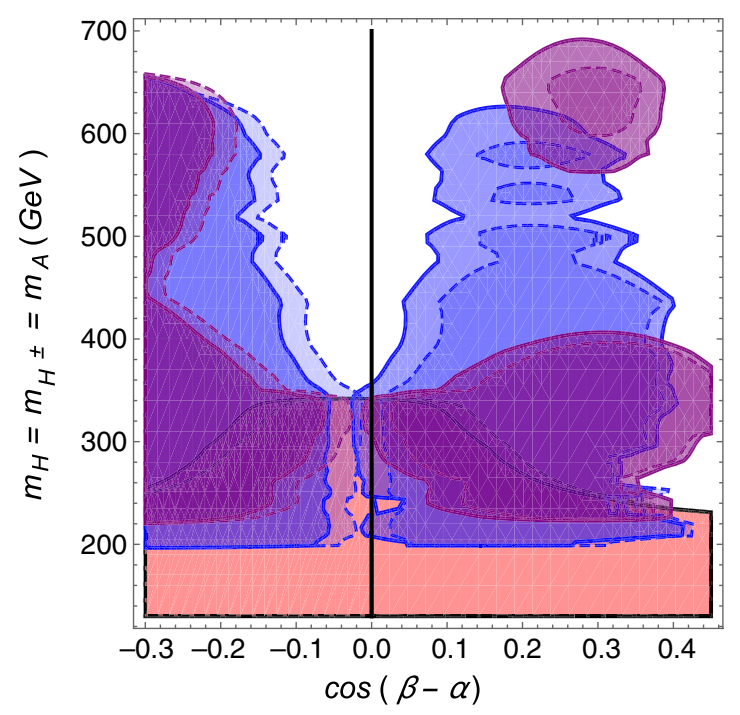

(a)

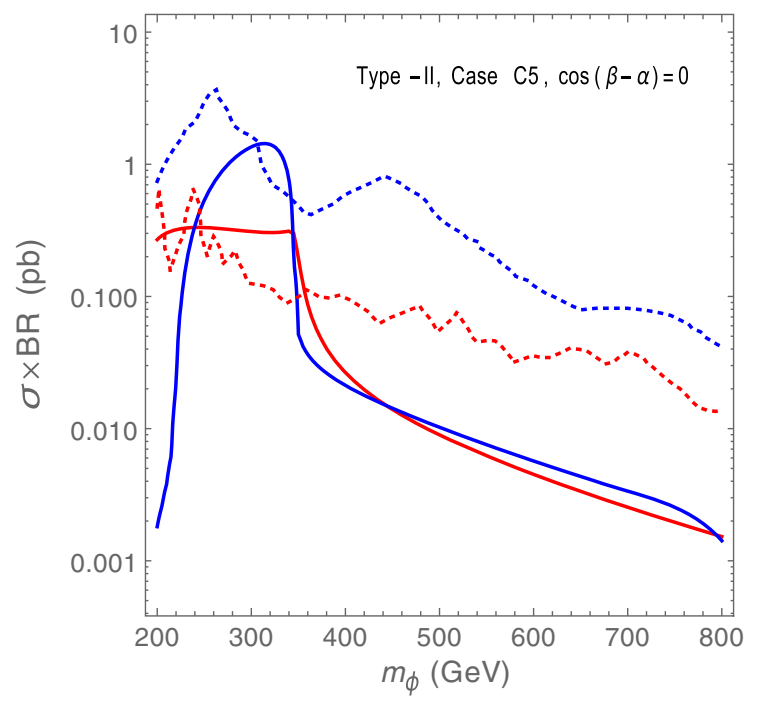

(b)

FIG. 5. The effect of the $\varphi^{4} D^{2}$ type of operators for $\mathbf{C 5}$ in a type-II 2HDM. (a) The blue regions with the dashed and solid boundaries are ruled out from $H \rightarrow Z Z$ [48], the pink regions with the dashed and solid boundaries are ruled out from $A \rightarrow \tau \tau$ [51], and the purple regions with the dashed and solid boundaries are ruled out from $A \rightarrow Z h$ [50] in a 2HDM and BP1 of 2HDMEFT, respectively. (b) The solid blue (red) line represents $\sigma \times \mathrm{Br}$ for $g g \rightarrow H \rightarrow Z Z$ [48] ( $g g \rightarrow A \rightarrow Z h$ [50]) at the alignment limit in the presence of the 6-dim operators mentioned in BP1. The experimental upper limits to the $\sigma \times \mathrm{Br}$ corresponding to the two processes are also shown as dotted lines in the same color.

become nonvanishing, ruling out a range of values of $m_{H}$ even at $\cos (\beta-\alpha)=0$. There is an overall leftward shift in the region ruled out by $A \rightarrow \tau \bar{\tau}$ which can be followed from Eqs. (2.7) and (4.2). For example, the values $m_{H} \sim 210-355 \mathrm{GeV}$ and $m_{A} \sim 300-340 \mathrm{GeV}$ can be excluded from $H \rightarrow Z Z$ and $A \rightarrow Z h$, respectively, at 95\% C.L. even in the alignment limit. In Fig. 5(b), we have shown the cross section of $H$ and $A$ via the gluon fusion times the branching ratios in the channels $Z Z$ and $Z h$ at the alignment limit in the presence of the higherdimensional operators. The value of $\sigma \times \mathrm{Br}$ for $H \rightarrow Z Z$ reaches $\sim 0.30 \mathrm{pb}$ in the range $m_{H}=200-344 \mathrm{GeV}$. For $A \rightarrow Z h$ it can reach up to $1.5 \mathrm{pb}$ in the range $m_{A} \sim 280-330 \mathrm{GeV}$. For a $2 \mathrm{HDM}$ at the tree level, such processes would not at all exist in the alignment limit.

It is to be noted that, according to Eq. (2.7), for a particular value of $|\cos (\beta-\alpha)|$ and $\tan \beta \gtrsim 1$, the $h b \bar{b}$ coupling multiplier is more pronounced in the negative direction of $\cos (\beta-\alpha)$, compared to the positive direction. This effect also propagates in the gluon-fusion cross section of $H$. Thus, the area on the $\cos (\beta-\alpha)-m_{A}$ plane ruled out by $g g \rightarrow H \rightarrow Z Z$ is larger on the negative $\cos (\beta-\alpha)$ direction. The relevant branching ratios of the heavy scalars have been presented in Appendix B.

In Fig. 6, we have shown the change in the excluded region from various searches of the new scalars on the $m_{H}-\tan \beta$ plane. Here, $\cos (\beta-\alpha)=0.1$ and $m_{A}=m_{H^{ \pm}}=m_{H}+$ $100 \mathrm{GeV}$. So, this is essentially a hierarchical mass scenario

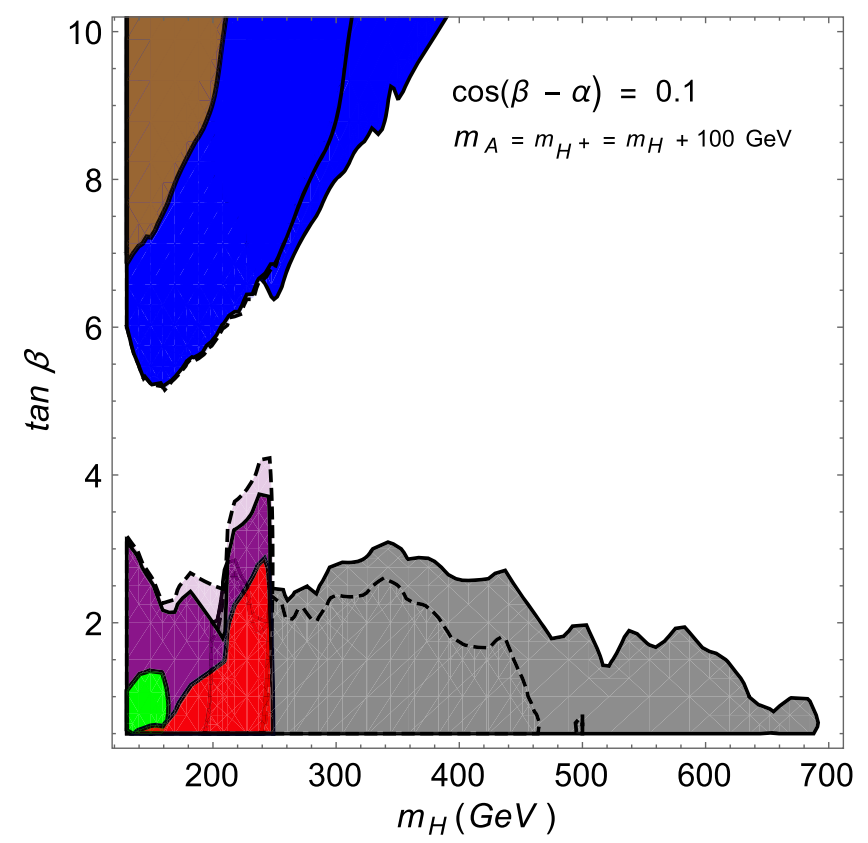

FIG. 6. The effect of the $\varphi^{4} D^{2}$ type of operators on the $m_{H}-$ $\tan \beta$ plane in a type-II 2 HDM. The regions are excluded from $g g \rightarrow H \rightarrow Z Z$ (gray) [48], $g g \rightarrow A \rightarrow Z H$ (purple) [50], $g g \rightarrow$ $A \rightarrow Z h$ (red) [50], gg $\rightarrow A \rightarrow \tau \bar{\tau}$ (green) [51], $b \bar{b} \rightarrow A \rightarrow \tau \bar{\tau}$ (brown) [51], and $b \bar{b} \rightarrow H \rightarrow \tau \bar{\tau}$ (blue) [51,52]. In the case of 2HDMEFT (dashed lines), the bounds from $A \rightarrow Z H$ and $H \rightarrow Z Z$ are different compared to a $2 \mathrm{HDM}$ at tree level (solid lines). 


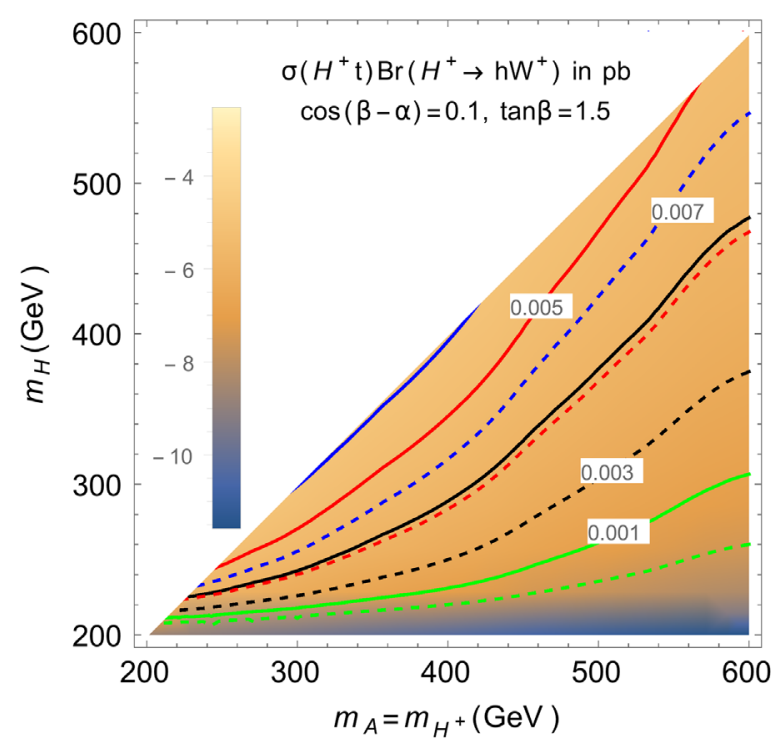

(a)

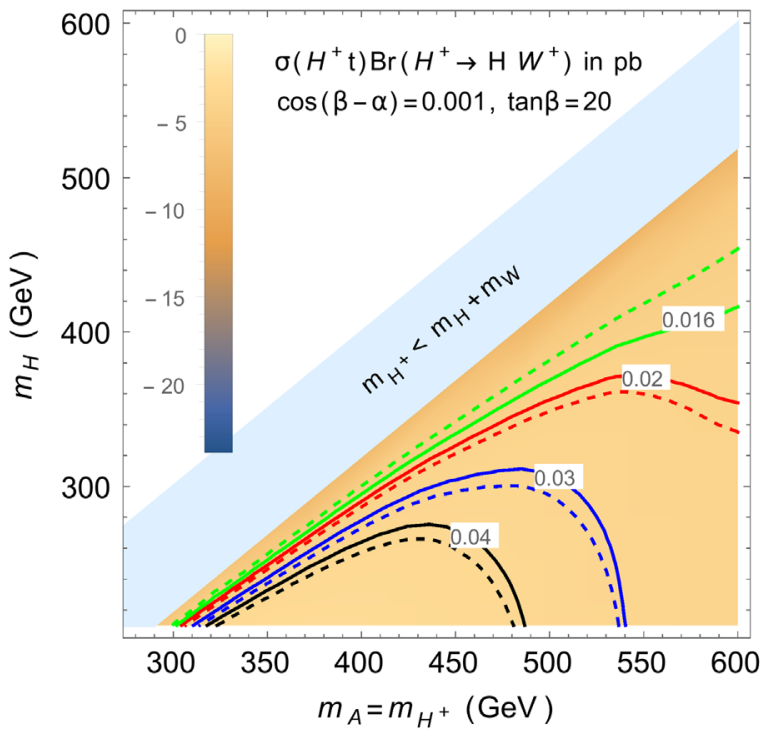

(c)

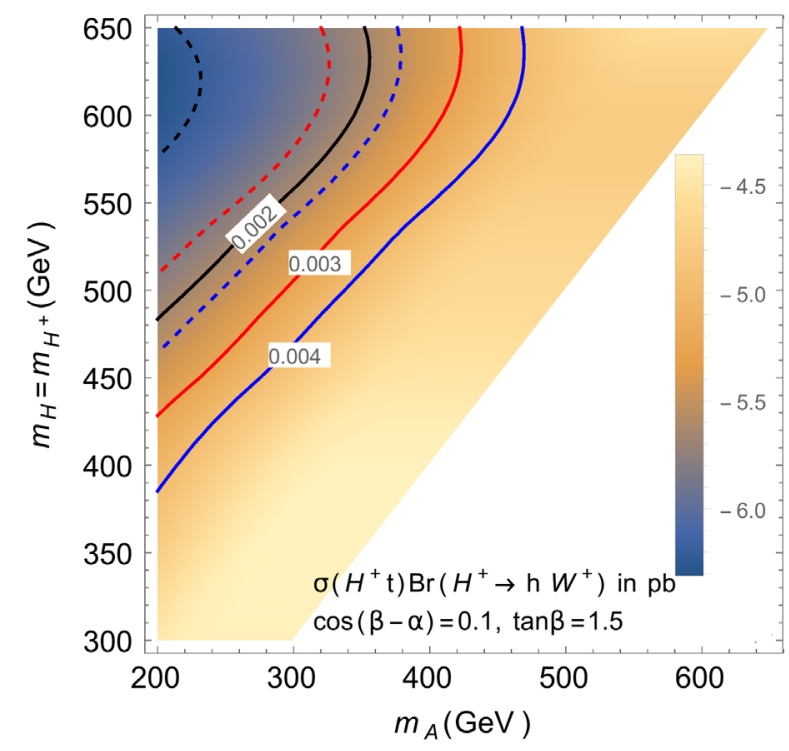

(b)

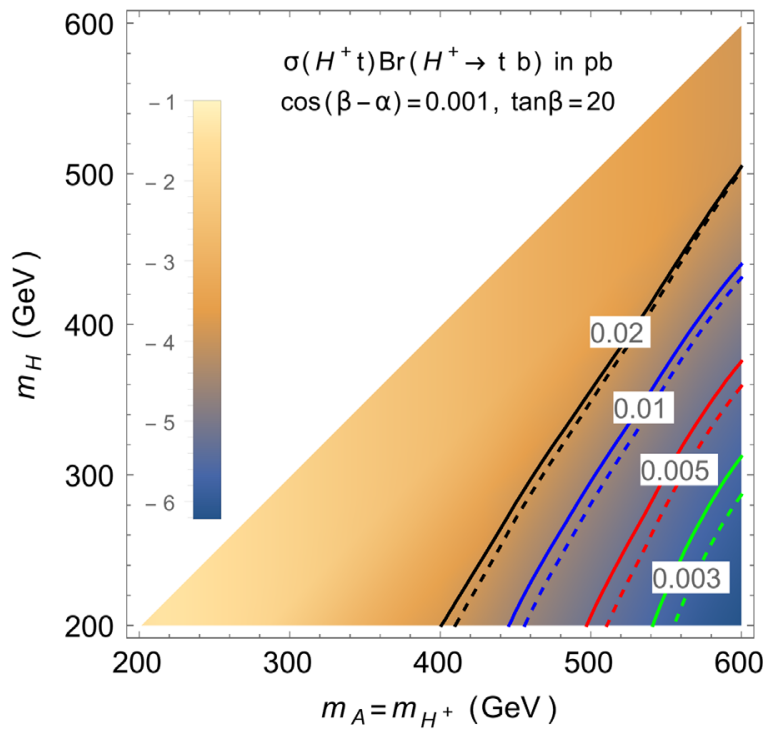

(d)

FIG. 7. The effect of 6-dim terms in a type-II $2 \mathrm{HDM}$ on (a) $\sigma\left(H^{ \pm} t\right) \operatorname{Br}\left(H^{ \pm} \rightarrow h W^{ \pm}\right)$for case $\mathbf{C 1}$, (b) $\sigma\left(H^{ \pm} t\right) \mathrm{Br}\left(H^{ \pm} \rightarrow h W^{ \pm}\right)$for case C4, (c) $\sigma\left(H^{ \pm} t\right) \operatorname{Br}\left(H^{ \pm} \rightarrow H W^{ \pm}\right)$for case $\mathbf{C 1}$, and (d) $\sigma\left(H^{ \pm} t\right) \operatorname{Br}\left(H^{ \pm} \rightarrow t \bar{b}\right)$ for case $\mathbf{C 1}$. The solid and dotted lines correspond to the same values of $\sigma \times \mathrm{Br}$ in a 2HDM at tree level and in BP1 of 2HDMEFT, respectively. The density plots depict the values of the corresponding $\sigma \times \mathrm{Br}$ in the log scale at the LHC with $\sqrt{s}=14 \mathrm{TeV}$.

where the exotic decay channel $A \rightarrow Z H$ plays a significant role at lower values of $\tan \beta$. For a type-II $2 \mathrm{HDM}$, both $h b \bar{b}$ and $h \tau \bar{\tau}$ couplings grow with increasing $\tan \beta$. Thus, in Fig. 6, higher values of $\tan \beta$ are mostly ruled out from the measurements of $b \bar{b} \rightarrow H \rightarrow \tau \bar{\tau}$. On introduction of the 6-dim terms, the region excluded from $H \rightarrow Z Z$ increases, whereas that from $A \rightarrow Z H$ shrinks. For instance, in 2HDMEFT the values $m_{H} \approx 464-686 \mathrm{GeV}$ can be ruled out for $\tan \beta \lesssim 1.5$. Moreover, the excluded region becomes larger in the direction of $\tan \beta$ for $m_{H} \approx 254-464 \mathrm{GeV}$.
So far, we have considered the phenomenology of only the neutral scalars. Now we comment on a few effects of the 6-dim terms in 2HDMEFT on the decay modes of the charged scalars. We calculate the production cross section of the charged scalar following Ref. [77] as was recommended in Ref. [78]. For $m_{H^{ \pm}}>m_{t}$, the key production channel of $H^{ \pm}$is through the process $p p \rightarrow H^{ \pm} t$. The $H^{ \pm} t b$ coupling multiplier depends on the value of $\tan \beta$ and the top and bottom quark masses. Following the $\tan \beta$ dependence of $\sigma\left(H^{ \pm} t\right)$, we rescale $\sigma\left(H^{ \pm} t\right)$ at $\tan \beta=30$ 
with the appropriate numerical factor to obtain the cross sections at $\tan \beta=1.5$ based on Figs. 3 and 10 of Ref. [77]. The traditional search channels of a charged Higgs boson consider the decays $H^{+} \rightarrow \bar{\tau} \nu_{\tau}, t \bar{b}$ [79-81], etc. Pertaining to different mass spectra of the 2HDM, the cascade decay channels with other Higgses as intermediate states can be interesting; the bounds on charged Higgs mass can be relaxed significantly $[58,82]$. In Fig. 7 , we have shown the contours of $\sigma\left(H^{ \pm} t\right) \operatorname{Br}\left(H^{ \pm} \rightarrow h W^{ \pm}\right)$in BP1 of 2HDMEFT for the 2HDM mass spectrum corresponding to cases $\mathbf{C 1}$ and $\mathbf{C 4}$ as discussed earlier. $\operatorname{Br}\left(H^{ \pm} \rightarrow H W^{ \pm}\right)$can change for the mass spectrum $\mathbf{C 1}$ and can be followed from Fig. 7(c). The decay channels consisting of SM fermions, such as $H^{+} \rightarrow t \bar{b}, \bar{\tau} \nu_{\tau}, c \bar{s}$, etc., become quite important at higher values of $\tan \beta$. In Fig. 7(d), we have shown the change in $\sigma \times \mathrm{Br}$ with the decay channel $H^{+} \rightarrow t \bar{b}$, though the value of $\sigma \times \mathrm{Br}$ in this channel is around one order smaller compared to the current LHC bound on such a process.

The coupling multiplier $\kappa_{H^{ \pm} t b} \propto\left(m_{t} \tan \beta P_{L}+m_{b}\right.$ $\left.\cot \beta P_{R}\right)$, and thus it reaches its minimum around $\tan \beta \sim 6-8$. Thus, the production cross section of $H^{ \pm}$ associated with a top quark becomes quite small for such values of $\tan \beta$. So we work only in scenarios when $\tan \beta \sim 1$ and $\tan \beta \sim \mathcal{O}(10)$. We have not considered the case of $m_{H^{ \pm}} \lesssim m_{t}$ when the key production mode of the charged scalar is $p p \rightarrow t \bar{t}$ with one of the tops in the final state decaying through $t \rightarrow b W^{+}$and another one via $\bar{t} \rightarrow \bar{b} H^{-}$. For these values of $m_{H^{ \pm}}$, bosonic decay channels of $H^{ \pm}$, such as $H^{ \pm} \rightarrow h W^{ \pm}, H W^{ \pm}$, are kinematically forbidden, unless one considers $m_{H}<m_{h}$, which is not the case for us.

As mentioned earlier, the coupling $H^{ \pm} h W^{\mp}$ vanishes at the alignment limit in a 2HDM. But in the presence of the 6-dim terms with the Wilson coefficients as in BP1 of 2HDMEFT, the channel $H^{ \pm} \rightarrow h W^{ \pm}$can become significant following Eqs. (4.2). For the mass spectrum in case $\mathbf{C 3}$, the cross section in the channel $\sigma\left(H^{ \pm} t\right) \operatorname{Br}\left(H^{ \pm} \rightarrow h W^{ \pm}\right)$can go up to $\sim 25 \mathrm{fb}$ for $m_{H^{ \pm}} \sim 250 \mathrm{GeV}$ at the LHC with $\sqrt{s}=14 \mathrm{TeV}$. The dependence of the cross section in this channel on $m_{H^{ \pm}}$for all four hierarchical mass spectra can be followed from Appendix C.

The couplings of $h(125)$ will be even more precisely measured in future experiments. For instance, the coupling multipliers $\kappa_{h \gamma \gamma}$ and $\kappa_{h W W}$ are to be measured with an accuracy of $\sim 5 \%-7 \%$ and $\sim 4 \%-6 \%$, respectively, at the HL-LHC with luminosity $\sim 3 \mathrm{ab}^{-1}$ [83]. It can push a 2HDM, especially the ones with type-II, -III, and -IV Yukawa couplings, further close to $\cos (\beta-\alpha)=0$. However, the contributions of dim- 6 terms to the signal strengths of $h(125)$ do not decrease with the same scale. As was also discussed in Ref. [32], even at the exact limit $\cos (\beta-\alpha)=0$, the effects of the dim- 6 terms in masking the true alignment limit can be rather significant. Thus, even at the limit when the couplings of $h(125)$ are exactly at par with the SM expectations, the heavier scalars are not decoupled from the rest of the particle spectrum. This remarkable feature can also be interpreted as the violation of the sum rules involving the couplings of the $C P$-even Higgses in a 2HDM extended with dim-6 operators. In this paper, we have demonstrated several cases where the cross sections of certain decay channels of the heavier scalars are significant in the presence of 6-dim terms at $\cos (\beta-\alpha)=0$. This leads to an interesting possibility of detecting the heavier scalars in these channels at the HL-LHC, even if $h(125)$ exactly resembles the SM Higgs.

\section{SUMMARY AND DISCUSSIONS}

In the context of the searches for new scalars at the LHC, it is an interesting possibility that the exotic scalars in a $2 \mathrm{HDM}$ exist below the $\mathrm{TeV}$ scale, pertaining to the so-called alignment-without-decoupling scenario. A study in 2HDMEFT becomes relevant in this case. Such an approach is appealing, because it allows us to study the constraints in the $2 \mathrm{HDM}$ parameter space while remaining agnostic about any new physics beyond the 2HDM. In this paper, we have confined our discussion to the bosonic operators of $2 \mathrm{HDMEFT}$. The changes in the constraints on the masses of the exotic scalars of a $2 \mathrm{HDM}$ are studied in the presence of 6-dim operators of type $\varphi^{4} D^{2}$, because the other bosonic operators are quite constrained from electroweak precision tests.

We consider both degenerate and hierarchical mass spectra of the new scalars in this purpose and show the changes in the constraints for all four Yukawa types. The theoretical constraints, such as stability, perturbativity, and unitarity, as well as the measurement of the oblique parameters, restrict the mass differences of such scalars. In light of that, one can narrow down four types of the mass spectrum in the hierarchical case. We notice that in a couple of such cases, dubbed as $\mathbf{C 1}$ and $\mathbf{C 2}$ in the text, the constraints on the $m_{A}-m_{H}$ plane can be significantly relaxed in the presence of certain 6-dim operators of type $\varphi^{4} D^{2}$. For example, in case C2 with $\cos (\beta-\alpha)=0$, $\tan \beta=1.5$, and type-I Yukawa coupling, the upper limit on $m_{H}$ reduces to $\sim 196 \mathrm{GeV}$ in BP1 of 2HDMEFT from $\sim 300 \mathrm{GeV}$, which is the case for a $2 \mathrm{HDM}$ at tree level. Such changes are always more pronounced for type-I and -IV 2HDMs compared to types II and III.

At $\cos (\beta-\alpha)=0$, processes such as $H \rightarrow Z Z, A \rightarrow Z h$, etc., vanish for a $2 \mathrm{HDM}$ at the tree level, which is not the case if dim-6 operators are present. A nonzero value for $\operatorname{Br}(H \rightarrow W W)$ reduces the value of $\operatorname{Br}(H \rightarrow b \bar{b})$, which brings down the cross section for the process $p p \rightarrow A \rightarrow$ $Z H(b \bar{b})$, thus relaxing the constraint on $m_{H}$. Such changes are not significant at higher values of $\tan \beta$ irrespective of the mass spectrum under consideration. This happens because the SM fermionic decay modes of the heavier scalars dominate for higher values of $\tan \beta$ and the appearance of new bosonic decay channels cannot change the key decay channels involving SM fermions significantly. 
For the degenerate case, we notice that, for our chosen benchmark scenario, the region excluded from the nonobservation of $H$ and $A$ becomes larger in 2HDMEFT compared to a $2 \mathrm{HDM}$ at the tree level. It is also seen that, as was discussed above, a certain mass range for $m_{H}\left(=m_{A}\right)$ is ruled out even for $\cos (\beta-\alpha)=0$ from processes like $H \rightarrow W W, A \rightarrow Z h$, which usually vanish in a $2 \mathrm{HDM}$ at tree level. We have also shown in Fig. 7 the change in $\sigma \times \mathrm{Br}$ for various decay channels of the charged scalar in 2HDMEFT compared to a $2 \mathrm{HDM}$ at the tree level at the LHC with $\sqrt{s}=14 \mathrm{TeV}$.

The key reason for the change in the constraints on $2 \mathrm{HDM}$ parameter space upon including dim-6 operators of type $\varphi^{4} D^{2}$ lies in the redefinition of the $C P$-even Higgs fields, $h$, and $H$. This way the coupling multipliers involving the $C P$-even scalars are rescaled compared to the $2 \mathrm{HDM}$ at the tree level and lead to a change in the branching ratios of all the processes which involve $h$ and $H$. It leads to the departure of the "true" alignment limit from its tree-level 2HDM counterpart, i.e., $\cos (\beta-\alpha)=0$. As the projected accuracy of the $h(125)$ coupling measurement at a future version of the LHC, such as the HL-LHC, is at the level $\lesssim 5 \%-6 \%, 2 \mathrm{HDMs}$ might get further pushed to the alignment limit. Thus, in the presence of dim- 6 operators, even if the couplings of $h(125)$ turn out to be completely aligned with the SM Higgs, the heavier scalars in a 2HDM with masses $\lesssim \mathrm{TeV}$ still do not decouple from the SM sector; i.e., their discovery might still be viable. As mentioned earlier, some cascade-type decay channels of the heavier scalars vanish at the alignment limit in the treelevel 2HDM. It implies that the discovery of a new scalar in such a channel would perhaps rule out the alignment limit in a $C P$-conserving $2 \mathrm{HDM}$. But if dim-6 operators are present, even if a new scalar is discovered in such channels, it will no longer rule out the alignment limit.

In the case of the discovery of the new Higgs(es), the verification of the sum rules involving their coupling multipliers can provide useful information about the nature of the extended Higgs sector. In 2HDMEFT, the redefinition of the $C P$-even Higgs fields due to $\varphi^{4} D^{2}$ operators also implies that the sum rules involving these scalars are modified in a certain way. We have discussed how the measurement of sum rules can help distinguish between various options beyond a $C P$-even $2 \mathrm{HDM}$.

If new scalars are discovered at the LHC in the near future, the correlation of their signal strengths in different channels will be important to determine the exact nature of the underlying scalar sector. In this context, 2HDMEFT can be an efficient framework in quantifying the departure from the tree-level $2 \mathrm{HDM}$ in various channels, providing an opportunity to narrow down the possible UV-complete scenarios.

\section{ACKNOWLEDGMENTS}

S. K. acknowledges T. Stefaniak for help regarding 2HDMC. This work is supported by the Department of Science and Technology, India via SERB Grant No. EMR/ 2014/001177 and DST-DAAD Grant No. INT/FRG/ DAAD/P-22/2018.

\section{APPENDIX A: FIELD REDEFINITION}

The redefinition of the physical $C P$-even neutral scalars in 2HDMEFT compared to a $2 \mathrm{HDM}$ at the tree level is given by Eq. (4.2) along with

$$
\begin{aligned}
x_{1}= & \frac{v^{2}}{f^{2}}\left(c_{H 1} c_{\beta}^{2} s_{\alpha}^{2}+c_{H 2} c_{\alpha}^{2} s_{\beta}^{2}+\frac{1}{8} c_{H 1 H 2} s_{2 \alpha} s_{2 \beta}+c_{H 12}\left(c_{\alpha}^{2} c_{\beta}^{2}+s_{\alpha}^{2} s_{\beta}^{2}-\frac{1}{4} s_{2 \alpha} s_{2 \beta}\right)\right. \\
& \left.+c_{H 1 H 12} c_{\beta} s_{\alpha}\left(s_{\alpha} s_{\beta}-\frac{1}{2} c_{\alpha} c_{\beta}\right)+c_{H 2 H 12} c_{\alpha} s_{\beta}\left(c_{\alpha} c_{\beta}-\frac{1}{2} s_{\alpha} s_{\beta}\right)\right), \\
x_{2}= & \frac{v^{2}}{f^{2}}\left(c_{H 1} c_{\beta}^{2} c_{\alpha}^{2}+c_{H 2} s_{\alpha}^{2} s_{\beta}^{2}+\frac{1}{8} c_{H 1 H 2} s_{2 \alpha} s_{2 \beta}+c_{H 12}\left(s_{\alpha}^{2} c_{\beta}^{2}+c_{\alpha}^{2} s_{\beta}^{2}-\frac{1}{4} s_{2 \alpha} s_{2 \beta}\right)\right. \\
& \left.+c_{H 1 H 12} c_{\beta} c_{\alpha}\left(c_{\alpha} s_{\beta}-\frac{1}{2} s_{\alpha} c_{\beta}\right)+c_{H 2 H 12} s_{\alpha} s_{\beta}\left(s_{\alpha} c_{\beta}-\frac{1}{2} c_{\alpha} s_{\beta}\right)\right), \\
y= & \frac{v^{2}}{f^{2}}\left(\frac{1}{2} c_{H 1} s_{2 \alpha} c_{\beta}^{2}-\frac{1}{2} c_{H 2} s_{2 \alpha} s_{\beta}^{2}-\frac{1}{8} c_{H 1 H 2} c_{2 \alpha} s_{2 \beta}-\frac{1}{2} c_{H 12}\left(c_{2 \beta} s_{2 \alpha}+\frac{1}{2} c_{2 \alpha} s_{2 \beta}\right)\right. \\
& \left.+\frac{1}{4} c_{H 1 H 12}\left(s_{2 \alpha} s_{2 \beta}-c_{2 \alpha} c_{\beta}^{2}\right)-\frac{1}{4} c_{H 2 H 12}\left(s_{2 \alpha} s_{2 \beta}+c_{2 \alpha} s_{\beta}^{2}\right)\right) .
\end{aligned}
$$




\section{APPENDIX B: BRANCHING RATIOS IN 2HDM VS 2HDMEFT}

The key branching ratios of $H$ and $A$ have been shown in Figs. 8 and 9 with type-I and -II Yukawas for mass spectra C1 and C2. When $m_{A(H)} \gtrsim 2 m_{t}$, the channel $A(H) \rightarrow t \bar{t}$ becomes viable in all the cases. While showing the branching ratios for $A$ we assume $m_{H}=m_{H^{ \pm}}=130 \mathrm{GeV}$, and while showing the same for $H$ we take $m_{A}=m_{H}+$ $100 \mathrm{GeV}$. For both these cases, we take $\cos (\beta-\alpha)=0$ and $\tan \beta=1.5 . \operatorname{Br}(A \rightarrow t \bar{t})$ attains values up to $\sim 0.4$ and $\sim 0.19$ for cases $\mathbf{C 1}$ and $\mathbf{C 2}$, respectively. In $\mathbf{C 2}$, $\operatorname{Br}\left(A \rightarrow H^{ \pm} W^{\mp}\right) \gtrsim 0.8$ for both type-I and -II 2HDMs, whereas this process is absent for the case $\mathbf{C 1}$. This leads to much lower values of $\operatorname{Br}(A \rightarrow Z H)$ in $\mathbf{C 2}$ compared to $\mathbf{C 1}$, which can be seen from Figs. 8(a) and 8(b). Hence, the nonobservation of $A \rightarrow Z H$ rules out a larger region of parameter space in the case $\mathbf{C 1}$ compared to $\mathbf{C} \mathbf{2}$ even in a tree-level 2HDM, which can be seen from Figs. 1 and 2. From Figs. 8(a) and 8(c), it can be seen that $\operatorname{Br}(A \rightarrow \tau \bar{\tau})$ is larger for a type-II $2 \mathrm{HDM}$ compared to type I. Thus, the nonobservation of $A \rightarrow \tau \bar{\tau}$ rules out a larger region for a type-II $2 \mathrm{HDM}$, as can be seen from, for example, Figs. 1(a) and 1(b). Figure 9(a) shows that $\operatorname{Br}(H \rightarrow W W)$ can attain values up to $\sim 0.5$ for a type-I $2 \mathrm{HDM}$ for case $\mathbf{C 1}$ at $\cos (\beta-\alpha)=0$ in the presence of a 6-dim term, whereas

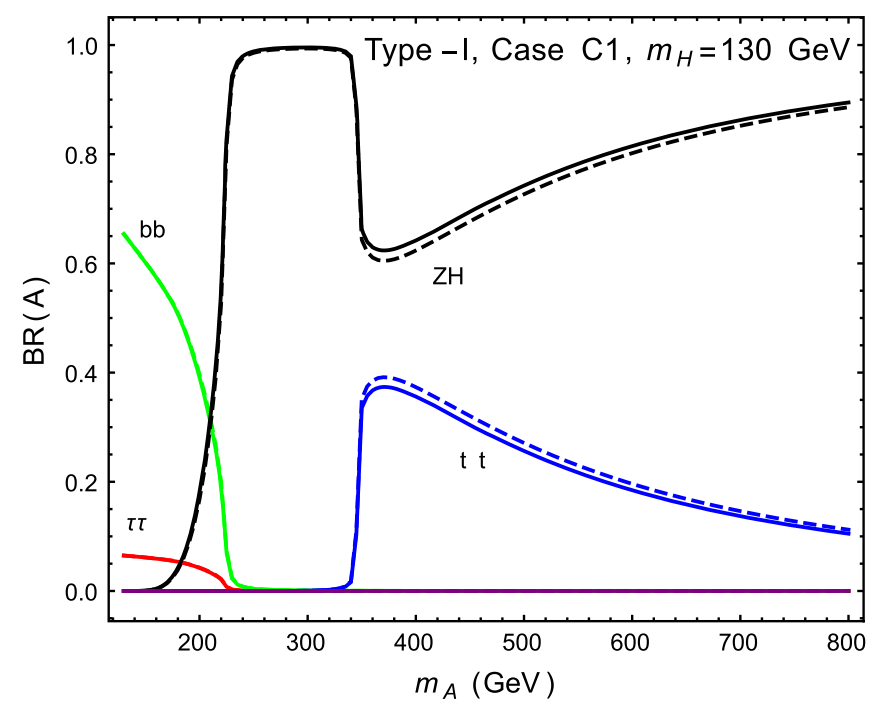

(a)

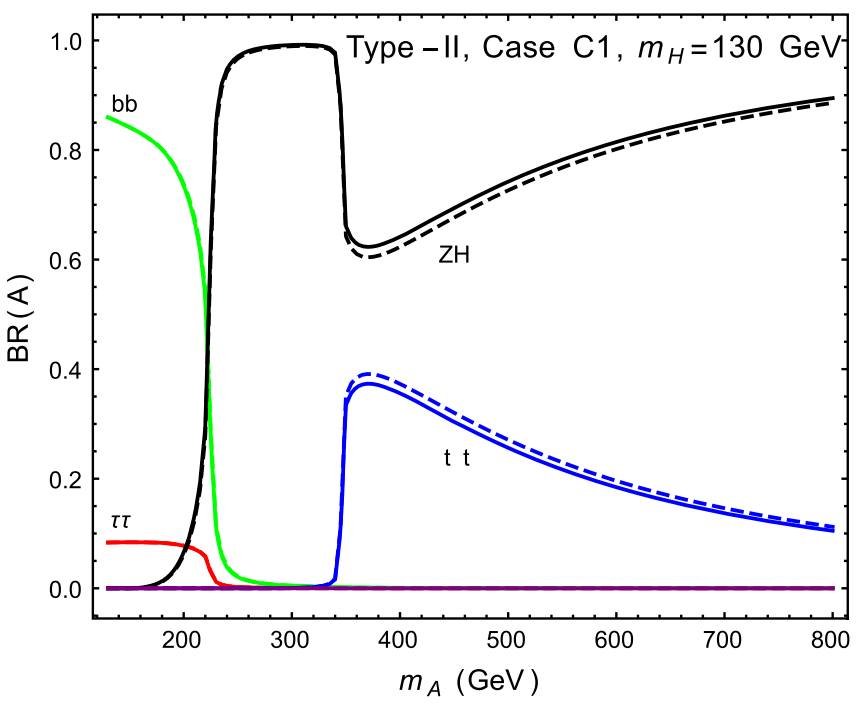

(c)

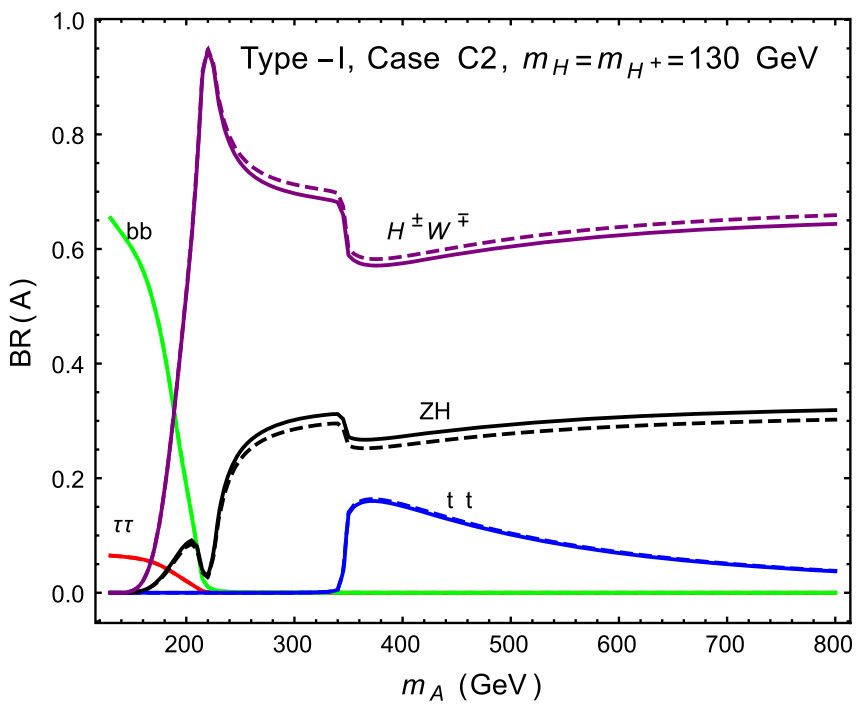

(b)

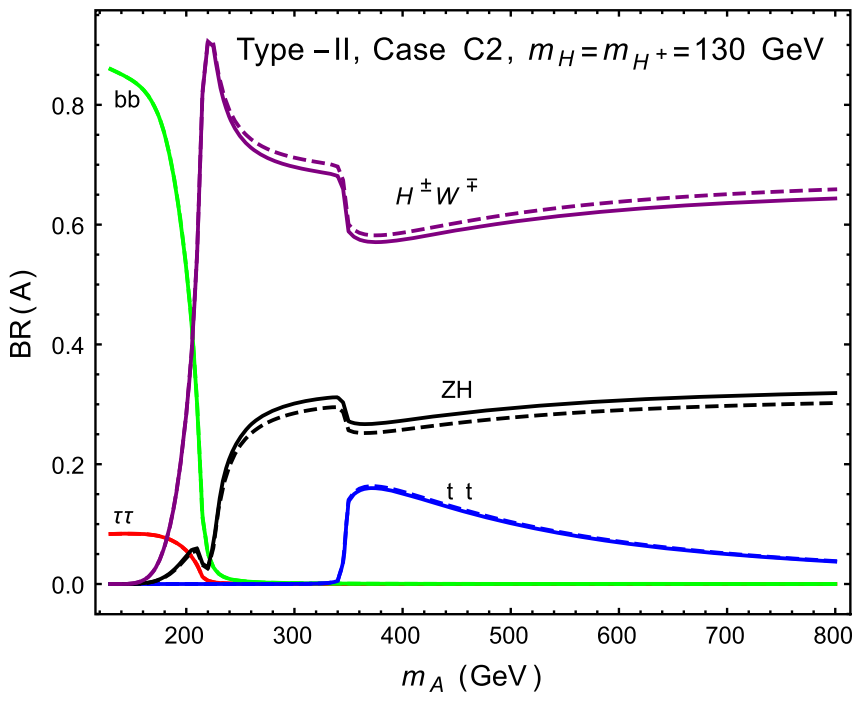

(d)

FIG. 8. The branching ratios of $A$ in various channels for the hierarchical cases for $\cos (\beta-\alpha)=0$ and $\tan \beta=1.5$. Dashed and solid lines represent the case of a $2 \mathrm{HDM}$ at tree level and BP1 of $2 \mathrm{HDMEFT}$, respectively. 


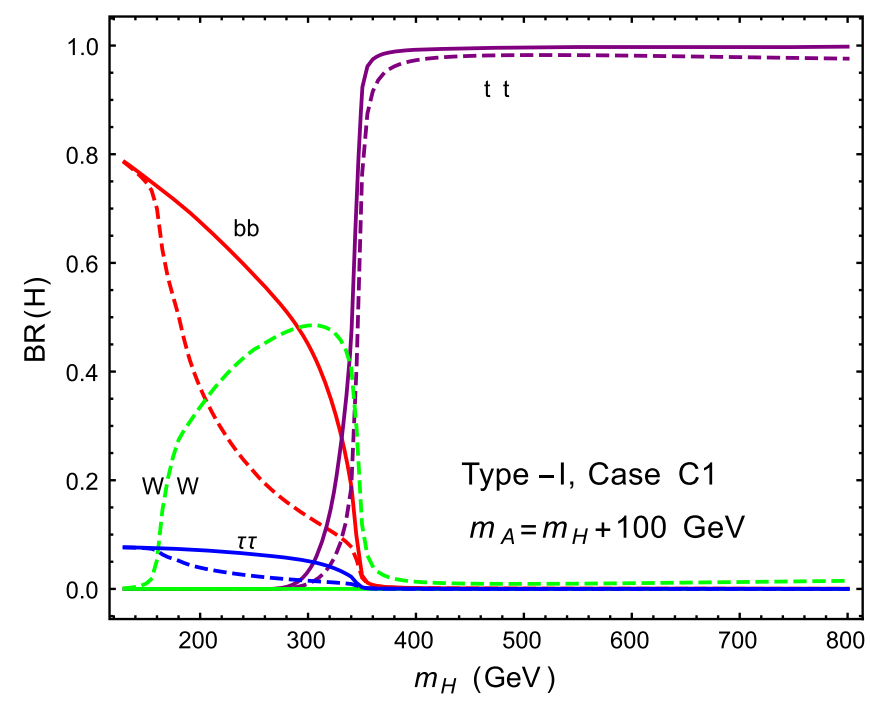

(a)

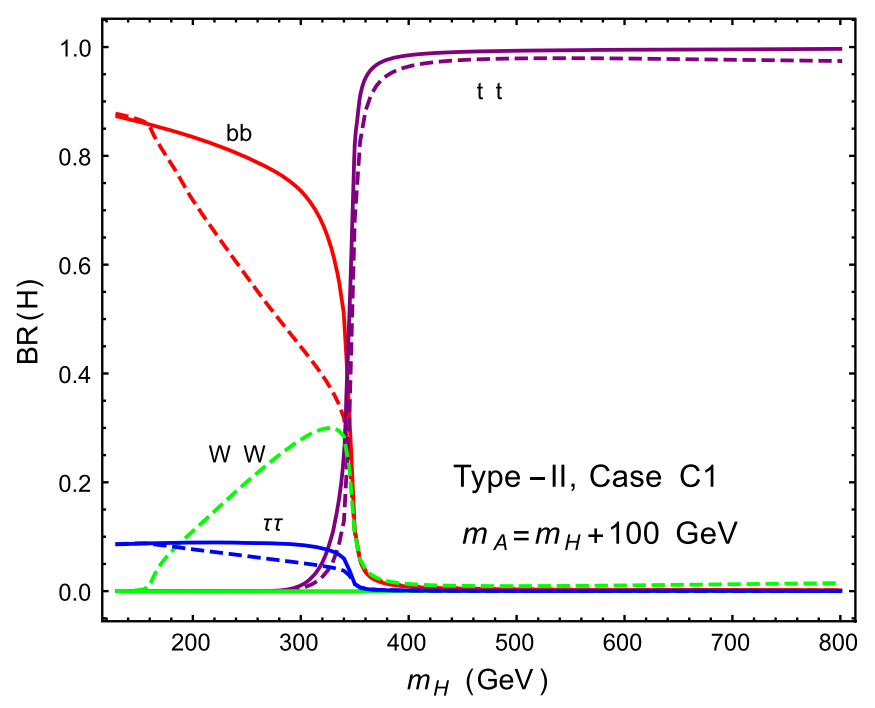

(b)

FIG. 9. The branching ratios of $H$ in various channels for the hierarchical case $\mathbf{C} \mathbf{1}$ for $\cos (\beta-\alpha)=0$ and tan $\beta=1.5$. Dashed and solid lines represent the case of a $2 \mathrm{HDM}$ at tree level and BP1 of 2HDMEFT, respectively.

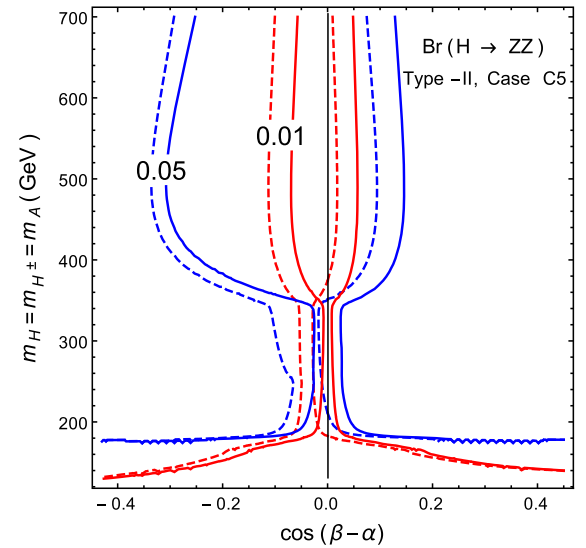

(a)

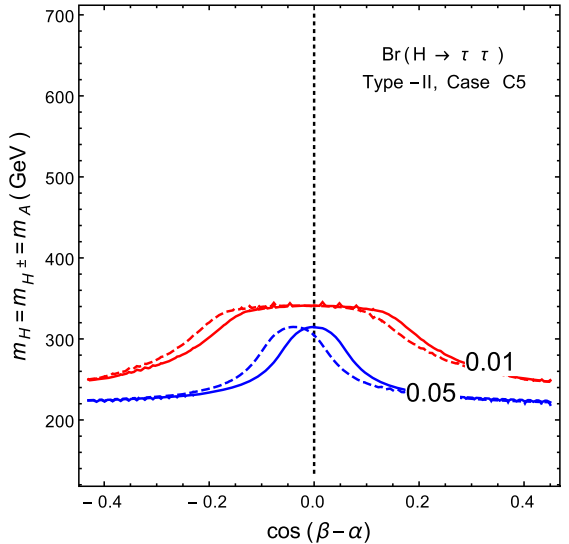

(b)

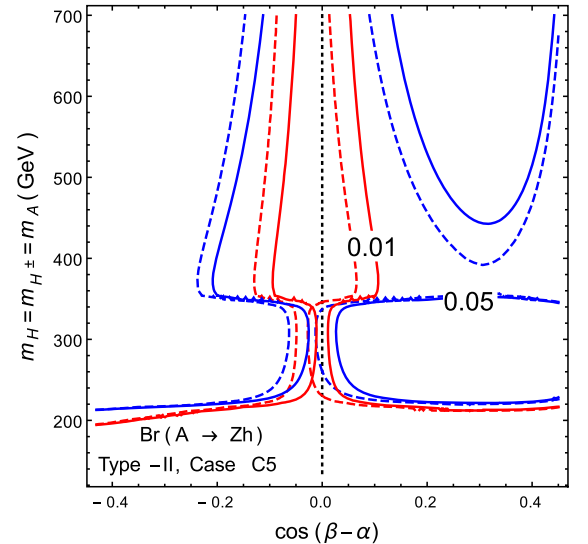

(c)

FIG. 10. The branching ratios of $H$ and $A$ in various channels which constrain the parameter space on the $\cos (\beta-\alpha)-m_{A}$ plane in the degenerate case $\mathbf{C 5}$ for a type-II 2HDM. In all three figures, the solid and dashed lines represent the corresponding branching ratios in a $2 \mathrm{HDM}$ at the tree level and BP1 of 2HDMEFT, respectively.

it is vanishing in a tree-level 2HDM. In contrary, as can be seen from Fig. 9(b), the corresponding value for a type-II $2 \mathrm{HDM}$ can only go up to $\sim 0.3$. The branching ratios of $H$ for case $\mathbf{C} 2$ are exactly the same as in the case $\mathbf{C} 1$. Such nonzero branching ratios of $H \rightarrow W W$ lead to a low value of $\operatorname{Br}(H \rightarrow b \bar{b})$ and eventually a lower value of $A \rightarrow Z H(b \bar{b})$ compared to a $2 \mathrm{HDM}$ at the tree level, which explains the relaxed constraints on $m_{H}$ as illustrated in Figs. 1 and 2 on the $m_{A}-m_{H}$ plane. Such branching ratios in type-III and -IV $2 \mathrm{HDMs}$ can also be followed from Figs. 8 and 9 along with Eq. (2.7), exploiting the patterns of couplings across Yukawa types. It can be seen that the constraints for a type-I Yukawa resemble that for type IV, whereas the constraints on a type-II $2 \mathrm{HDM}$ are similar to that in the type-III case. The branching ratios for $H \rightarrow$ $Z Z, \tau \bar{\tau}$ and $A \rightarrow Z h$ in the degenerate benchmark scenario C5 has been shown in Fig. 10.

\section{APPENDIX C: $\boldsymbol{H}^{ \pm} \rightarrow \boldsymbol{h} \boldsymbol{W}^{ \pm}$CROSS SECTION $\mathrm{AT} \cos (\beta-\alpha)=0$}

The cross sections in the channel $p p \rightarrow H^{ \pm} \rightarrow h W^{ \pm}$ for different hierarchical mass spectra and type-II Yukawa couplings have been presented in Fig. 11. For $\cos (\beta-\alpha)=0$, in a $2 \mathrm{HDM}$ at the tree level, such a process is absent. But in the presence of the 6-dim operators, the 


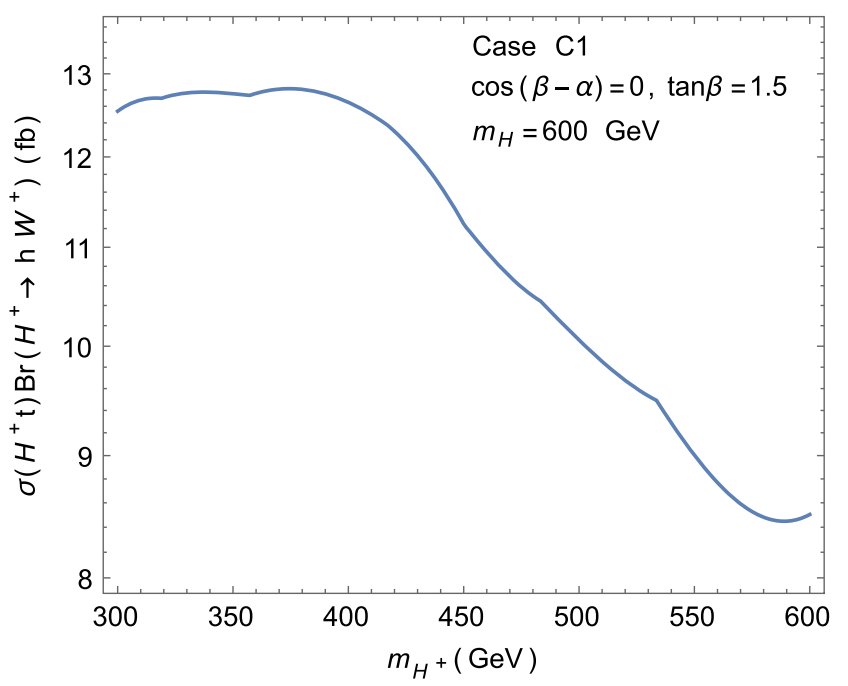

(a)

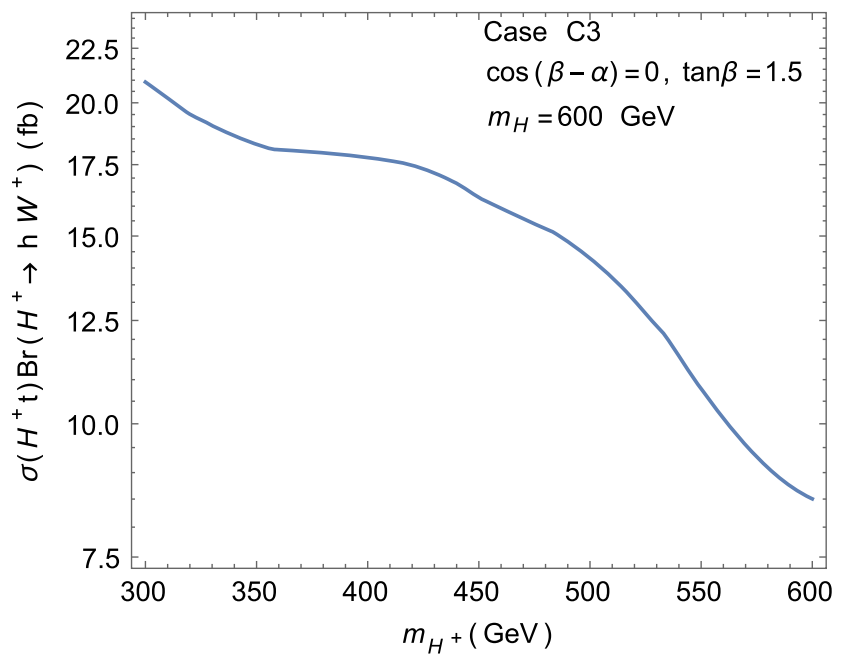

(c)

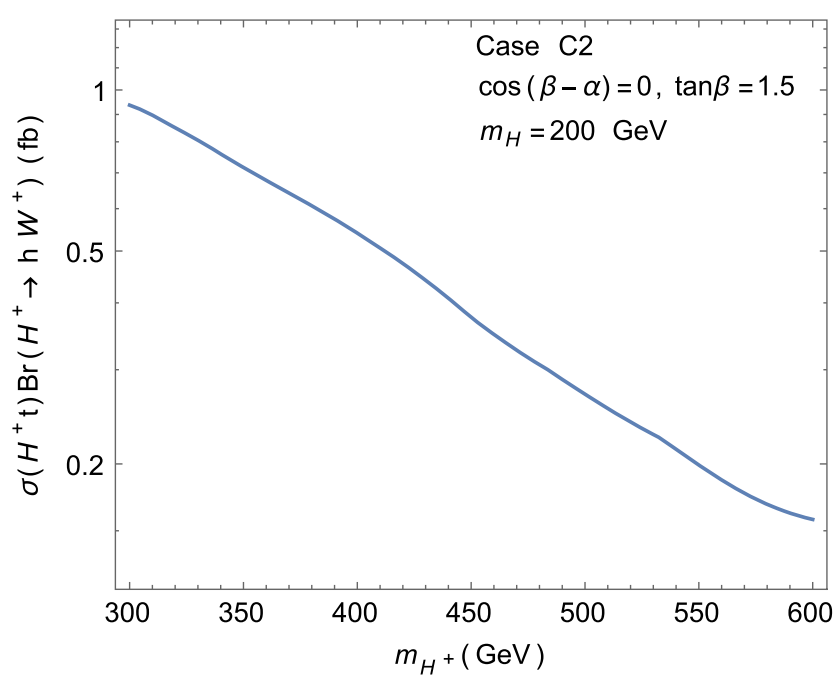

(b)

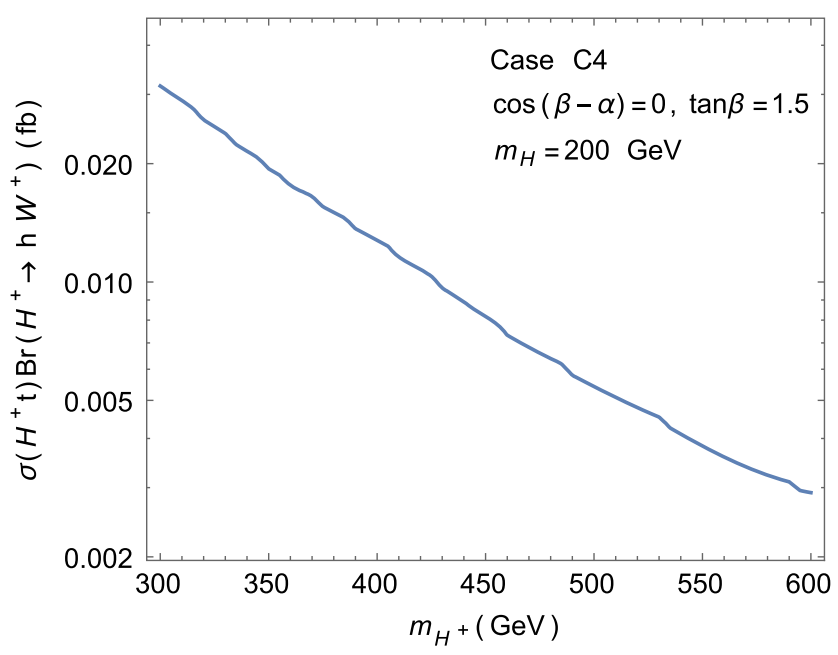

(d)

FIG. 11. $\sigma\left(H^{ \pm} t\right) \operatorname{Br}\left(H^{ \pm} \rightarrow h W^{ \pm}\right)$in the alignment limit, in the presence of the 6-dim terms at the LHC with $\sqrt{s}=14$ TeV in a type-II 2HDM.

cross section for this process can go up to $\sim 25 \mathrm{fb}$ depending on the mass spectrum of heavy scalars in the 2HDM. It can be noticed that the cross section in this channel attains significantly higher values for the cases C1, C2, and C3 compared to $\mathbf{C 4}$. For $\mathbf{C 4}$, the channel $H^{ \pm} \rightarrow A W^{ \pm}$becomes kinematically accessible, thus lowering the value of $\operatorname{Br}\left(H^{ \pm} \rightarrow h W^{ \pm}\right)$, leading to a lesser cross section compared to the other cases.
[1] G. Aad et al. (ATLAS Collaboration), Phys. Lett. B 716, 1 (2012).

[2] S. Chatrchyan et al. (CMS Collaboration), Phys. Lett. B 716, 30 (2012).

[3] P. Fayet, Phys. Lett. B 64, 159 (1976).

[4] J. Mrazek, A. Pomarol, R. Rattazzi, M. Redi, J. Serra, and A. Wulzer, Nucl. Phys. B853, 1 (2011).
[5] S. De Curtis, S. Moretti, K. Yagyu, and E. Yildirim, Eur. Phys. J. C 77, 513 (2017).

[6] M. Schmaltz, D. Stolarski, and J. Thaler, J. High Energy Phys. 09 (2010) 018.

[7] J. H. Yu, J. High Energy Phys. 12 (2016) 143.

[8] M. Trodden, Rev. Mod. Phys. 71, 1463 (1999). 
[9] J. M. Cline, K. Kainulainen, and M. Trott, J. High Energy Phys. 11 (2011) 089.

[10] A. Crivellin, C. Greub, and A. Kokulu, Phys. Rev. D 86, 054014 (2012).

[11] A. Crivellin, D. Müller, and C. Wiegand, J. High Energy Phys. 06 (2019) 119.

[12] E. Ma, Phys. Rev. D 73, 077301 (2006).

[13] M. D. Campos, D. Cogollo, M. Lindner, T. Melo, F. S. Queiroz, and W. Rodejohann, J. High Energy Phys. 08 (2017) 092.

[14] R. Barbieri, L. J. Hall, and V. S. Rychkov, Phys. Rev. D 74, 015007 (2006).

[15] H. Georgi and D. V. Nanopoulos, Phys. Lett. B 82, 95 (1979).

[16] J. F. Gunion and H. E. Haber, Phys. Rev. D 67, 075019 (2003).

[17] A. Delgado, G. Nardini, and M. Quiros, J. High Energy Phys. 07 (2013) 054.

[18] M. Carena, I. Low, N. R. Shah, and C. E. M. Wagner, J. High Energy Phys. 04 (2014) 015.

[19] J. Bernon, J. F. Gunion, H. E. Haber, Y. Jiang, and S. Kraml, Phys. Rev. D 92, 075004 (2015).

[20] J. Bernon, J. F. Gunion, H. E. Haber, Y. Jiang, and S. Kraml, Phys. Rev. D 93, 035027 (2016).

[21] H. E. Haber, arXiv:1401.0152.

[22] H. E. Haber, arXiv:1805.05754.

[23] B. Grzadkowski, H. E. Haber, O. M. Ogreid, and P. Osland, J. High Energy Phys. 12 (2018) 056.

[24] J. L. Diaz-Cruz, J. Hernandez-Sanchez, and J. J. Toscano, Phys. Lett. B 512, 339 (2001).

[25] A. Crivellin, M. Ghezzi, and M. Procura, J. High Energy Phys. 09 (2016) 160.

[26] S. Karmakar and S. Rakshit, J. High Energy Phys. 10 (2017) 048.

[27] M. Chala, G. Durieux, C. Grojean, L. de Lima, and O. Matsedonskyi, J. High Energy Phys. 06 (2017) 088.

[28] Y. Kikuta, Y. Okada, and Y. Yamamoto, Phys. Rev. D 85, 075021 (2012).

[29] Y. Kikuta and Y. Yamamoto, Eur. Phys. J. C 76, 297 (2016).

[30] N. Fonseca, R. Zukanovich Funchal, A. Lessa, and L. Lopez-Honorez, J. High Energy Phys. 06 (2015) 154.

[31] A. Banerjee, G. Bhattacharyya, and N. Kumar, Phys. Rev. D 99, 035028 (2019).

[32] S. Karmakar and S. Rakshit, J. High Energy Phys. 09 (2018) 142.

[33] I. F. Ginzburg and M. Krawczyk, Phys. Rev. D 72, 115013 (2005).

[34] A. W. El Kaffas, P. Osland, and O. M. Ogreid, Nonlinear Phenom. Complex Syst. 10, 347 (2007).

[35] S. L. Glashow and S. Weinberg, Phys. Rev. D 15, 1958 (1977).

[36] E. A. Paschos, Phys. Rev. D 15, 1966 (1977).

[37] J. Gu, H. Li, Z. Liu, S. Su, and W. Su, J. High Energy Phys. 12 (2017) 153.

[38] G. C. Dorsch, S. J. Huber, K. Mimasu, and J. M. No, Phys. Rev. D 93, 115033 (2016).

[39] P. S. Bhupal Dev and A. Pilaftsis, J. High Energy Phys. 12 (2014) 024; 11 (2015) 147(E).

[40] A. Pilaftsis, Phys. Rev. D 93, 075012 (2016).
[41] D. Das, U. K. Dey, and P. B. Pal, Phys. Rev. D 96, 031701 (2017).

[42] K. Benakli, Y. Chen, and G. Lafforgue-Marmet, Eur. Phys. J. C 79, 172 (2019).

[43] S. Pramanick and A. Raychaudhuri, J. High Energy Phys. 01 (2018) 011.

[44] N. Craig, J. Galloway, and S. Thomas, arXiv:1305.2424.

[45] P. M. Ferreira, R. Guedes, J. F. Gunion, H. E. Haber, M. O. P. Sampaio, and R. Santos, arXiv:1410.1926.

[46] T. Modak, J. C. Romao, S. Sadhukhan, J. P. Silva, and R. Srivastava, Phys. Rev. D 94, 075017 (2016).

[47] P. M. Ferreira, J. F. Gunion, H. E. Haber, and R. Santos, Phys. Rev. D 89, 115003 (2014).

[48] ATLAS Collaboration, CERN Report No. ATLAS-CONF2017-055, 2017.

[49] A. M. Sirunyan et al. (CMS Collaboration), Phys. Lett. B 788, 7 (2019).

[50] ATLAS Collaboration, CERN Report No. ATLAS-CONF2016-015, 2016.

[51] ATLAS Collaboration, CERN Report No. ATLAS-CONF2017-050, 2017.

[52] CMS Collaboration, CERN Report No. CMS-PAS-HIG-14029, 2015.

[53] G. C. Dorsch, S. J. Huber, K. Mimasu, and J. M. No, Phys. Rev. Lett. 113, 211802 (2014).

[54] G. C. Dorsch, S. J. Huber, and J. M. No, J. High Energy Phys. 10 (2013) 029.

[55] C. Gao, M. A. Luty, M. Mulhearn, N. A. Neill, and Z. Wang, Phys. Rev. D 97, 075040 (2018).

[56] M. Bauer, M. Carena, and K. Gemmler, J. High Energy Phys. 11 (2015) 016.

[57] F. Kling, A. Pyarelal, and S. Su, J. High Energy Phys. 11 (2015) 051.

[58] B. Coleppa, F. Kling, and S. Su, J. High Energy Phys. 12 (2014) 148.

[59] F. Kling, J. M. No, and S. Su, J. High Energy Phys. 09 (2016) 093.

[60] A. Adhikary, S. Banerjee, R. K. Barman, and B. Bhattacherjee, arXiv:1812.05640.

[61] F. Kling, H. Li, A. Pyarelal, H. Song, and S. Su, J. High Energy Phys. 06 (2019) 031.

[62] J.-M. Gerard and M. Herquet, Phys. Rev. Lett. 98, 251802 (2007).

[63] S. de Visscher, J. M. Gerard, M. Herquet, V. Lemaitre, and F. Maltoni, J. High Energy Phys. 08 (2009) 042.

[64] W. Grimus, L. Lavoura, O. M. Ogreid, and P. Osland, J. Phys. G 35, 075001 (2008).

[65] W. Grimus, L. Lavoura, O. M. Ogreid, and P. Osland, Nucl. Phys. B801, 81 (2008).

[66] H. E. Haber and D. O'Neil, Phys. Rev. D 83, 055017 (2011).

[67] H. J. He, N. Polonsky, and S. f. Su, Phys. Rev. D 64, 053004 (2001).

[68] G. Abbiendi et al. (ALEPH, DELPHI, L3, OPAL, and LEP Collaborations), Eur. Phys. J. C 73, 2463 (2013).

[69] S. Schael et al. (ALEPH, DELPHI, L3, and OPAL Collaborations and LEP Working Group for Higgs Boson Searches), Eur. Phys. J. C 47, 547 (2006).

[70] F. Mahmoudi and O. Stäl, Phys. Rev. D 81, 035016 (2010).

[71] O. Eberhardt, U. Nierste, and M. Wiebusch, J. High Energy Phys. 07 (2013) 118. 
[72] M. Misiak and M. Steinhauser, Eur. Phys. J. C 77, 201 (2017).

[73] T. Han, T. Li, S. Su, and L. T. Wang, J. High Energy Phys. 11 (2013) 053.

[74] D. Eriksson, J. Rathsman, and O. Stäl, Comput. Phys. Commun. 181, 189 (2010).

[75] S. De Curtis, S. Moretti, K. Yagyu, and E. Yildirim, Phys. Rev. D 94, 055017 (2016).

[76] R. V. Harlander, S. Liebler, and H. Mantler, Comput. Phys. Commun. 184, 1605 (2013).

[77] M. Flechl, R. Klees, M. Kramer, M. Spira, and M. Ubiali, Phys. Rev. D 91, 075015 (2015).
[78] S. Heinemeyer et al. (LHC Higgs Cross Section Working Group), arXiv:1307.1347.

[79] M. Aaboud et al. (ATLAS Collaboration), Phys. Lett. B 759, 555 (2016).

[80] CMS Collaboration, CERN Report No. CMS-PAS-HIG-16031, 2016.

[81] ATLAS Collaboration, CERN Report No. ATLAS-CONF2016-089, 2016.

[82] A. Arhrib, R. Benbrik, and S. Moretti, Eur. Phys. J. C 77, 621 (2017).

[83] ATLAS Collaboration, CERN Report No. ATL-PHYSPUB-2018-054, 2018. 